\title{
TLR3 deficiency impairs spinal cord synaptic transmission, central sensitization, and pruritus in mice
}

\author{
Tong Liu, ${ }^{1}$ Temugin Berta, ${ }^{1}$ Zhen-Zhong Xu, ${ }^{1}$ Chul-Kyu Park, ${ }^{1}$ Ling Zhang, ${ }^{1}$ Ning Lü, ${ }^{1}$ Qin Liu, ${ }^{2}$ \\ Yang Liu, ${ }^{3}$ Yong-Jing Gao, ${ }^{1}$ Yen-Chin Liu, ${ }^{1}$ Qiufu Ma, ${ }^{3}$ Xinzhong Dong, ${ }^{2}$ and Ru-Rong Ji1 \\ ${ }^{1}$ Sensory Plasticity Laboratory, Pain Research Center, Department of Anesthesiology, Perioperative and Pain Medicine, Brigham and Women's Hospital \\ and Harvard Medical School, Boston, Massachusetts, USA. ${ }^{2}$ The Solomon H. Snyder Department of Neuroscience, Center for Sensory Biology, \\ School of Medicine, Johns Hopkins University, Baltimore, Maryland, USA. ${ }^{3}$ Dana-Farber Cancer Institute and Department of Neurobiology,
} Harvard Medical School, Boston, Massachusetts, USA.

\begin{abstract}
Itch, also known as pruritus, is a common, intractable symptom of several skin diseases, such as atopic dermatitis and xerosis. TLRs mediate innate immunity and regulate neuropathic pain, but their roles in pruritus are elusive. Here, we report that scratching behaviors induced by histamine-dependent and -independent pruritogens are markedly reduced in mice lacking the $T l r 3$ gene. TLR3 is expressed mainly by small-sized primary sensory neurons in dorsal root ganglions (DRGs) that coexpress the itch signaling pathway components transient receptor potential subtype V1 and gastrin-releasing peptide. Notably, we found that treatment with a TLR3 agonist induces inward currents and action potentials in DRG neurons and elicited scratching in WT mice but not $\mathrm{Tlr} 3^{-/-}$mice. Furthermore, excitatory synaptic transmission in spinal cord slices and long-term potentiation in the intact spinal cord were impaired in $\mathrm{Tlr}^{-/-}$mice but not $\mathrm{Tlr} 7^{-/-}$mice. Consequently, central sensitization-driven pain hypersensitivity, but not acute pain, was impaired in $\mathrm{Tlr} 3^{-/-}$mice. In addition, TLR3 knockdown in DRGs also attenuated pruritus in WT mice. Finally, chronic itch in a dry skin condition was substantially reduced in $\mathrm{Tlr} 3^{-/-}$mice. Our findings demonstrate a critical role of TLR3 in regulating sensory neuronal excitability, spinal cord synaptic transmission, and central sensitization. TLR3 may serve as a new target for developing anti-itch treatment.
\end{abstract}

\section{Introduction}

Itch, also known as pruritus, is an unpleasant cutaneous sensation that evokes desire or reflex of scratching behavior, which is distinct from pain that elicits withdrawal reflex (1). Like pain, itch serves as a self-protective/warning system in the normal conditions (1). However, chronic itch is recurrent and intractable and greatly reduces the life quality of patients with skin diseases (e.g., atopic dermatitis, contact dermatitis, allergic contact dermatitis, and xerosis), liver diseases (e.g., cholestasis), kidney diseases (e.g., uremia), and metabolic disorders (e.g., diabetes) (2). Although itch is transiently relieved by scratching, itch-scratch cycles often result in further skin damage that exacerbates the problem. Antihistamines are normally used for itch relief but many chronic itchy conditions are resistant to them (3).

TLRs mediate innate immune responses via recognition of pathogen-associated molecular patterns (4). While TLR3 recognizes double-stranded RNA (dsRNA), TLR7 detects single-stranded RNA or imidazoquinoline derivatives (4). It has been shown that microglia recognize dsRNA-producing virus in the CNS via activation of TLR3 (5). TLR3 also mediates West Nile virus (a mosquito-borne RNA flavivirus) entry into the brain, causing lethal encephalitis (6). Of interest, TLR2, TLR3, and TLR4 contribute to the development of nerve injury-induced neuropathic pain by inducing glial activation and expression of proinflammatory cytokines in the spinal cord (7-9). Although TLRs are typically expressed by immune and glial cells, increasing evidence indicates that primary sensory neu-

Authorship note: Tong Liu and Temugin Berta contributed equally to this work. Conflict of interest: The authors have declared that no conflict of interest exists. Citation for this article: J Clin Invest. 2012;122(6):2195-2207. doi:10.1172/JCI45414. rons also express TLRs (e.g., TLR3, TLR4, TLR9) (10-12). But the functions of these TLRs in sensory neurons are largely unknown. Recently, we demonstrated that TLR7 is functionally expressed in transient receptor potential cation channel subfamily $\mathrm{V}$ member 1positive (TRPV1-positive) nociceptors and contributed to histamine-independent pruritus, without affecting mechanical, thermal, inflammatory, and neuropathic pain (13). In this study, we tested the hypothesis that TLRs play distinct roles in pain and itch. We found that TLR 3 is essential for eliciting both histamine-dependent and -independent pruritus, whereas TLR7 is only partially required for producing histamine-independent itch. We also demonstrated that chronic itch following dry skin-induced dermatitis is abrogated in $\mathrm{Tlr}^{-/-}$mice. By contrast, acute pain after thermal, mechani$\mathrm{cal}$, and chemical stimulation is normal in $\mathrm{Tlr}^{-/-}$mice. Further, we found that TLR3 ligands are sufficient to induce inward currents and action potentials in dorsal root ganglion (DRG) neurons and further elicit scratching behaviors in mice.

Central sensitization, i.e., hyperactivity of spinal cord dorsal horn neurons after tissue injury or persistent nociceptive input (e.g., C-fiber activation), is believed to cause hypersensitivity to pain (14) and itch (15). Spinal cord long-term potentiation (LTP) is a unique form of synaptic plasticity (16) and central sensitization (14). In this study, we also examined the role of TLR3 in central sensitization and spinal cord synaptic plasticity. We found that spinal LTP was induced in $\mathrm{Tl}_{\mathrm{r}} \mathrm{T}^{-/}$mice but not $\mathrm{Tlr}^{-/-}$mice. Consequently, behavioral expression of central sensitization, i.e., the formalin-induced second-phase pain and the capsaicin-induced secondary mechanical hyperalgesia (17), was impaired in $\mathrm{Tlr}^{-/-}$mice. Thus, TLR3 might control itch via novel neuronal mechanisms, including peripheral mechanisms 
A Rotarod

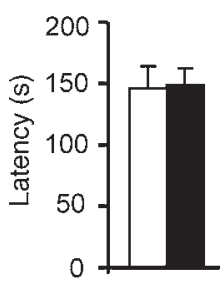

B Hargreaves

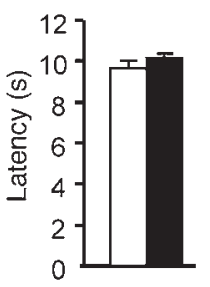

C

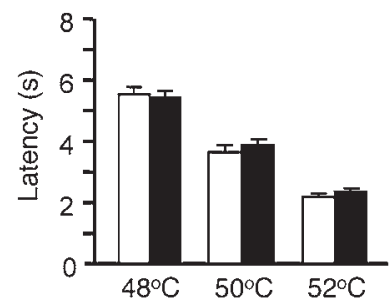

Formalin

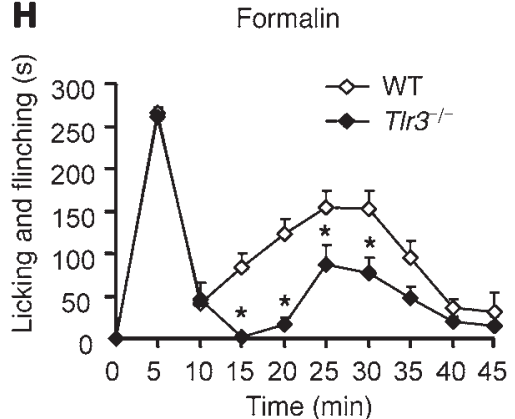

D von Frey

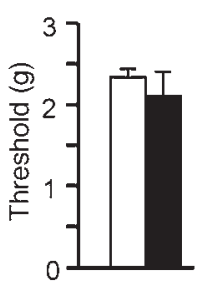

F

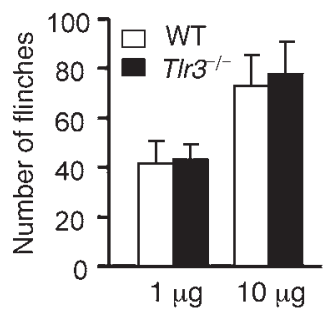

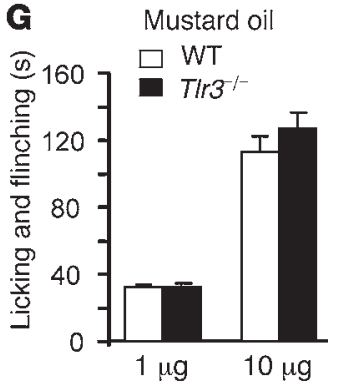

Figure 1
I $1^{\text {si }}$ mechanical hypersensitivity after capsaicin

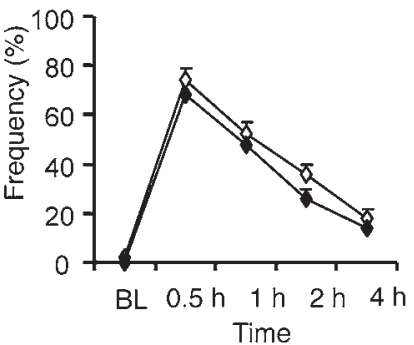

$2^{\text {nd }}$ mechanical hypersensitivity after capsaicin

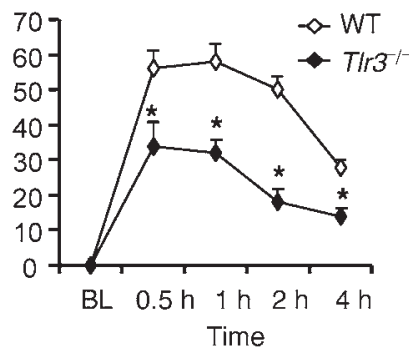

Intact acute pain but impaired central sensitization in TIr3-/- mice. (A) Motor function assessed by recording the falling latency in a Rotarod test

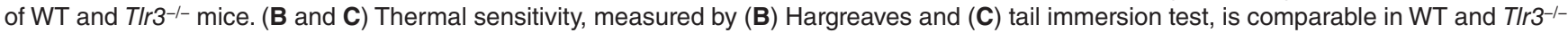
mice. (D and E) Mechanical sensitivity, assessed by (D) von Frey test and (E) Randall-Selitto test, is indistinguishable in WT and TIr3-/- mice. (F and G) Acute spontaneous pain assessed by the number of flinches or duration of licking and flinching behaviors after intraplantar injection of (F) capsaicin and (G) mustard oil in WT and T/r3-/- mice. (H) Spontaneous pain in the second phase (10-45 minutes) but not in the first phase (0-10 minutes) in the formalin test is decreased in $T / 3^{-/-}$mice. (I) Primary and secondary mechanical hypersensitivity after intraplantar injection of capsaicin $(5 \mu \mathrm{g})$, assessed by percentage response (frequency) to a von Frey filament $(0.16 \mathrm{~g})$, in WT and T/r $3^{-/-}$mice. TIr $3^{-/-}$mice have intact primary mechanical hyperalgesia but impaired secondary mechanical hyperalgesia. BL, baseline. * $P<0.05$, compared with WT mice, Student's $t$ test; $n=5-9$ mice for each group. All the data are mean \pm SEM.

(altering excitability of primary sensory neurons) and central mechanisms (modulating synaptic plasticity) as well as classic nonneuronal/immune mechanisms in the pathological conditions.

\section{Results}

Tlr $3^{-/}$mice display intact acute nociceptive pain but impaired central sensitization-driven pain. To determine the role of TLR 3 in acute pain sensation, we performed multiple behavioral tests of acute pain in WT mice and mice lacking TLR3 (Tlr3 $3^{-/}$mice) (18). Consistent with previous reports (18), we did not observe any gross anatomical defects in $\operatorname{Tlr}^{-/-}$mice (Supplemental Figure 1A; supplemental material available online with this article; doi:10.1172/ JCI45414DS1). The rotarod test revealed that the falling latency of $\mathrm{Tr}^{-/-}$mice was similar to that of WT mice, indicating there is no motor impairment in $T l r 3^{-/-}$mice (Figure 1A). Acute thermal sensitivity, as assessed by radiant heat (Hargreaves; Figure 1B) and hot water immersion (Figure 1C), and innocuous and noxious mechanical sensitivity, as evaluated by von Frey filament (Figure 1D) and the Randall-Selitto test (Figure 1E), respectively, were also indistinguishable in $\mathrm{Tlr}^{-/-}$and WT mice (Figure 1, B-E). Further, $\mathrm{Tlr}^{-/-}$mice showed normal spontaneous flinching/licking behaviors in response to the TRPV1 agonist capsaicin (Figure 1F) and the transient receptor potential cation channel, member ankyrin 1 (TRPA1) agonist mustard oil (Figure 1G).

Next, we examined formalin-induced (5\%) biphasic spontaneous pain in $\mathrm{Tlr}^{-1-}$ and WT mice. While the first-phase response is a result of acute activation of peripheral nociceptors, the second-phase response is attributed to activity-dependent central sensitization and ongoing afferent input (14). Notably, the second-phase responses but not the first-phase responses were significantly decreased in $\mathrm{Tlr}^{-/-}$ mice (Figure 1H; $P<0.05$, compared with WT mice, Student's $t$ test). Additionally, capsaicin-induced secondary but not primary mechanical hyperalgesia was reduced in $\mathrm{Tlr}^{-/-}$mice (Figure 1I; $P<0.05$, compared with WT mice, Student's $t$ test). Together, these results suggest that TLR3 is essential for central sensitization-driven pain hypersensitivity but not required for acute nociceptive pain.

Tlr $3^{-/-}$mice exhibit deficiency in both bistamine-dependent and -independent itch. We examined the scratching behaviors in $\mathrm{Tlr}^{-/-}$and WT mice after intradermal injection of histamine-dependent and -independent pruritic agents into the nape of the neck. While compound 48/80 induces histamine-dependent itch, via histamine release from mast cells, chloroquine (CQ; an anti-malarial drug) induces histamine-independent itch via activation of sensory neuron-specific GPCR MrgprA3 (19). Compared with that of WT mice, $\mathrm{Tlr}^{-/-}$mice exhibited a dramatic reduction in scratching behaviors evoked by compound 48/80 (Figure 2A; $P<0.05$, 2-way repeatedmeasures ANOVA) and CQ (Figure 2B; $P<0.05$, 2-way repeatedmeasures ANOVA). We did not find any difference when comparing sexes in the compound 48/80-induced scratching when testing the age-matched male and female $T / r 3^{-/-}$mice (data not shown). Spinal c-Fos expression was widely used as a functional marker of spinal neuronal activation after pruritic stimuli (20). c-Fos expression in dorsal horn neurons, induced either by compound 48/80 or CQ, was significantly decreased in $\mathrm{Tlr}^{-/-}$mice (Figure 2C; 


\section{A}

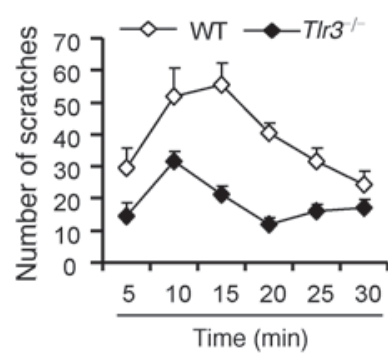

C
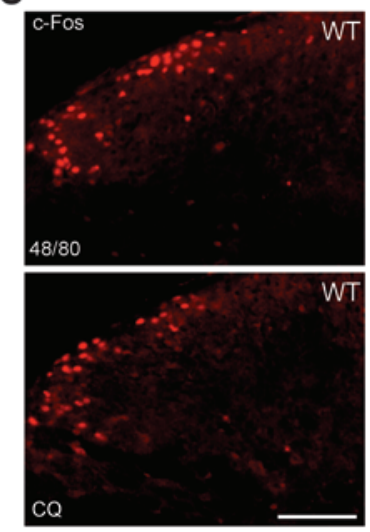
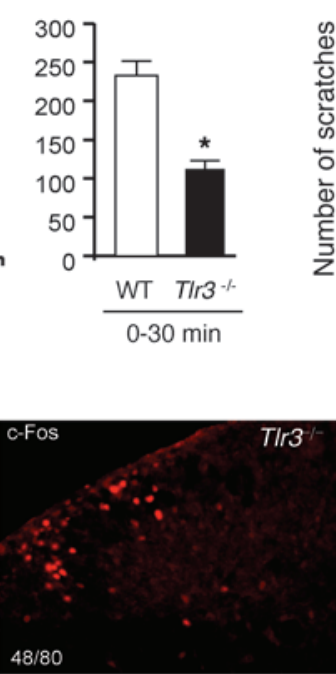

Tir3
B

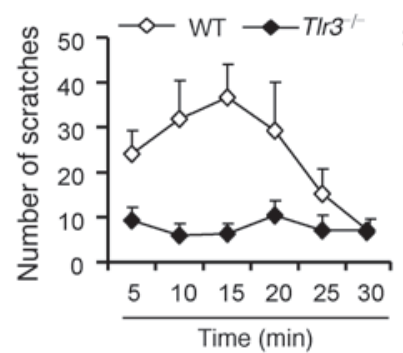

Compound $48 / 80$

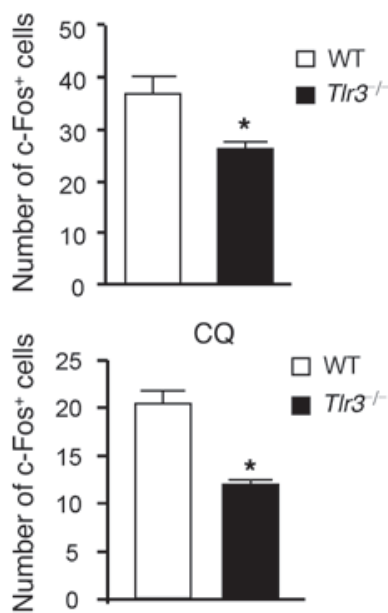

\section{Figure 2}

Impaired scratching behaviors and reduced c-Fos expression in the spinal cords in TIr $3^{-/-}$mice. (A and B) Scratches in every 5 minutes (left) and 0-30 minutes (right) induced by intradermal injection of $50 \mu \mathrm{l} \mathrm{compound} \mathrm{48/80} \mathrm{(100} \mu \mathrm{g})$ and CQ $(200 \mu \mathrm{g})$. Note a reduction of both histaminergic (compound 48/80) and nonhistaminergic (CQ) itch in TIr3-l- mice. ${ }^{\star} P<0.05$, Student's $t$ test; $n=11-13$ mice for each group. Mean \pm SEM. Twoway repeated-measures ANOVA analysis also shows a significant difference in the time course of compound 48/80- and CQ-induced scratching between the 2 groups $(P<0.05)$. (C) c-Fos-like immunoreactivity in the dorsal horn of the cervical spinal cord in WT and T/r3--- mice 2 hours after intradermal injection of compound $48 / 80(48 / 80)$ or CQ. Right panels show the number of c-Fos-positive neurons in the dorsal horn. Scale bars, $100 \mu \mathrm{m}$. ${ }^{*} P<0.05$, Student's $t$ test; $n=4-6$ mice. All the data are mean \pm SEM.

$P<0.05$, compared with WT mice, Student's $t$ test). These data suggest that both the pruritogen-induced itch behaviors and spinal neuronal activation were impaired in $\mathrm{Tlr}^{-/-}$mice.

We also tested additional histaminergic pruritogens, including histamine, histamine-trifluoromethyl-toluidine (HTMT, a histamine $\mathrm{H} 1$ receptor agonist), and 4-methylhistamine (4-MeHA, a histamine $\mathrm{H} 4$ receptor agonist). Scratching elicited by these pruritogens was significantly reduced in $\operatorname{Tlr}^{-1-}$ mice (Supplemental Figure 1, B-D; $P<0.05$, 2-way repeated-measures ANOVA). Further, scratching induced by endothelin-1 and serotonin, as well as nonhistaminergic pruritogens SLIGRL-NH2 (PAR2 agonist) $(21,22)$ and trypsin, was abrogated in $\mathrm{Tlr}^{-/-}$mice (Supplemental Figure 1, E-H; $P<0.05$, 2-way repeated-measures ANOVA). Finally, we tested scratching by using a low concentration of formalin (0.6\%) (23) and the TLR7 agonist imiquimod, which was shown to induce robust itch in mice $(13,24)$. Both formalin- and imiquimod-induced scratches were substantially reduced in $\mathrm{Tlr}^{-/-}$mice (Supplemental Figure 1, I and J; $P<0.05$, 2-way repeated-measures ANOVA).

Together, our analyses of scratching during a 30-minute period further confirmed that all forms of itch mentioned above were substantially decreased in $T l r 3^{-/-}$mice (Supplemental Figure $1 \mathrm{~K}$;
$P<0.05$, Student's $t$ test). Thus, TLR3 is indispensable for the full expression of acute itch behaviors in mice, regardless of the histamine dependency.

TLRs are involved differently in pain and itch in a cheek model. To further determine the distinct roles of TLRs in pain and itch, we used a recently developed cheek model that can distinguish pain versus itch $(25,26)$, as indicated by distinct pain-like wiping by forelimbs and itch-like scratching by the hind $\operatorname{limbs}(25,26)$. $\operatorname{Tr}^{-/-}$mice displayed a dramatic reduction in the compound 48/80- and CQinduced scratching $(P<0.05$, Student's $t$ test) but not the wiping (Figure 3A), confirming a role of TLR3 in itch but not acute nociception. Interestingly, $\operatorname{Tl} 7^{-/-}$mice only showed a partial reduction in CQ-induced scratching (Figure 3B; $P<0.05$, Student's $t$ test) but no changes in the compound 48/80- and CQ-induced wiping and the compound 48/80-induced scratching (Figure 3B), in support of our previous observation that TLR7 is only involved in histamine-independent itch (13).

TLR3 is expressed in a subset of DRG neurons. TLR3 was shown to be expressed by most embryonic DRG neurons during development (27). We further characterized TLR3 expression in postnatal DRG neurons using several different methods. 
A

Compound $48 / 80$

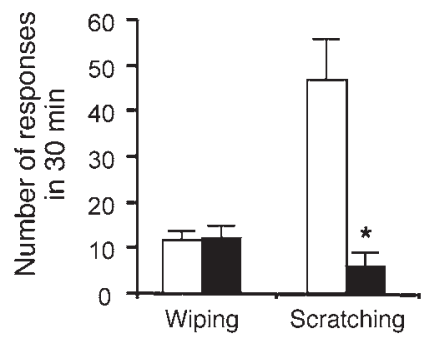

B Compound 48/80

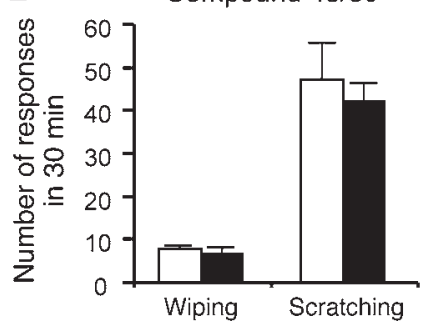

$\mathrm{CQ}$

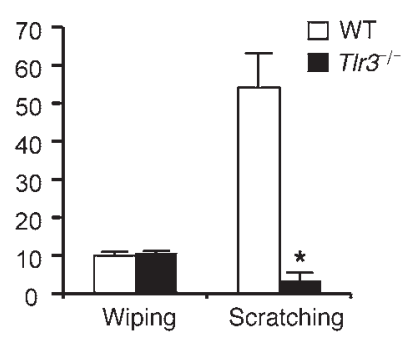

$\mathrm{CQ}$

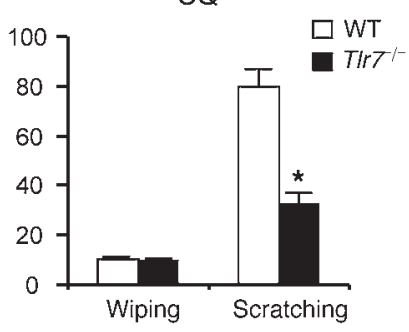

\section{Figure 3}

Distinct role of TLRs in regulating pain and itch in a mouse cheek model. Intradermal injection of compound 48/80 (50 $\mu \mathrm{g})$ and CQ $(100 \mu \mathrm{g})$ in the cheek induces either pain-like wiping by forelimbs or itch-like scratching by the hind limb. (A) Substantial reduction of compound 48/80- and CQ-induced scratching behaviors in TIr $3^{-1-}$ mice. (B) $\mathrm{CQ}$-induced but not compound $48 / 80$-induced scratching is reduced in $T / r 7^{-/-}$mice. Note that wiping behavior induced by compound $48 / 80$ or CQ is normal in both TIr3 ${ }^{-/-}$and $T / r 7^{-I-}$ mice. ${ }^{\star} P<0.05$, Student's $t$ test; $n=5-8$ mice. All the data are mean \pm SEM.

(a) RT-PCR analysis revealed that Tlr3 mRNA was expressed in DRG, spinal cord, brain, and spleen tissues of adult mice (Supplemental Figure 2A). (b) In situ hybridization showed that Tlr 3 mRNA was expressed by approximately $25 \%$ of DRG neurons (468 out of 1,849 neurons), mainly in small-sized neurons and some medium-sized neurons (Supplemental Figure 2B). (c) Single-cell RT-PCR analysis, conducted selectively in small-sized DRG neurons, further revealed that TLR3 and TLR7 were expressed by distinct but overlapping populations of small-sized DRG neurons (Figure 4A). Approximately 40\%, $50 \%$, and $70 \%$ of small neurons expressed TLR3, gastrin-releasing peptide (GRP), and TRPV1, respectively (Figure 4B). Notably, all TLR $3^{+}$neurons expressed TRPV1 and GRP (Figure 4B). (d) Immunohistochemistry further confirmed that TLR3 was highly colocalized with GRP (Figure 4C). TLR3 ${ }^{+}$and $\mathrm{GRP}^{+}$neurons also exhibited similar size distribution patterns (Figure 4D). (e) Immunocytochemistry validated that all TLR $3^{+}$neurons coexpressed TRPV1 (Figure 4E) but not neurofilament 200 (NF200), a marker for myelinated A-fiber neurons (Figure 4E). Given the unique roles of TRPV1- and GRP-expressing neurons in itch signaling $(28,29)$, the distinct localization of TLR3 in GRP/TRPV1-expressing neurons (Figure 4F) would provide a cellular basis for the involvement of TLR3 in pruritus.

TLR3 agonist poly (I:C) induces inward currents and action potentials in DRG neurons and also elicits scratching in mice in a TLR3-dependent manner. To further define whether TLR3 expressed by DRG neurons is functional, we examined DRG neuronal responses to a synthetic TLR3 agonist, poly (I:C) (PIC), in vitro. Whole-cell patch-clamp recording revealed that PIC elicited dose-dependent inward currents (200-1,000 ng/ml; Figure 5, A and B) in capsaicin-sensitive DRG neurons (Figure 5, A-C). Notably, PIC failed to induce inward currents in $\mathrm{Tlr}^{-/-}$mice (Figure 5, A and B). Single-cell RT-PCR analysis confirmed that all PIC-responsive neurons ( $40 \%$ of the recorded small-sized neurons) indeed expressed TLR3. Conversely, TLR3-negative neurons failed to respond to PIC (Figure 5C). Thus, the TLR3 expression in DRG neurons correlates well with the TLR3 function (PIC sensitivity). Additionally, current-clamp recordings demonstrated that PIC could elicit robust action potentials in capsaicin-sensitive DRG neurons of WT mice but not $\mathrm{Tlr}^{-/-}$mice (Figure 5D), suggesting that TLR3 activation can directly excite DRG neurons.

To correlate DRG neuronal activation with mouse behaviors, we also tested the PIC-induced itching. Intradermal injection of PIC induced dose-dependent scratching behaviors in WT mice (Figure 5E; $P<0.05$, 1-way ANOVA). Strikingly, PIC-induced scratching was completely abolished in $\mathrm{Tlr}^{-3^{--}}$mice (Figure 5F; $P<0.05$, Student's $t$ test).

We also observed TLR3 immunoreactivity in the substance Pexpressing (SP-expressing) peptidergic axons of the sciatic nerve (Supplemental Figure 3A) as well as in the skin nerve terminals (Supplemental Figure 3, B-D), indicating that TLR3 can be transported from the DRG cell bodies to the peripheral axons, in which TLR3 could detect endogenous or exogenous ligands. Although TLR3 is highly colocalized with TRPV1 in DRG neurons, PICinduced inward currents were only mildly reduced in $\operatorname{Tr} p v 1^{1 /-}$ mice $(19.0 \% \pm 8.2 \%$ reduction, $P<0.05$, Student's $t$ test, $n=8-15$ neurons). PIC-induced scratching was unaltered in the $\operatorname{Trp} v 1^{-/-}$mice compared with that in WT mice (Figure 5F).

To explore possible endogenous ligands of TLR3, we extracted total RNAs from mouse brain tissues and stimulated DRG neurons with the RNAs. Patch-clamp recordings showed that the extracted total RNAs were sufficient to induce dose-dependent inward currents in the dissociated small-sized DRG neurons that also responded to PIC and capsaicin (Figure 5, G and H). However, total RNAs failed to induce inward currents in DRG neurons of Tlr $3^{-/-}$mice (Figure 5, G and H). Thus, the total RNAs (presumably dsRNAs) can activate DRG neurons via TLR3.

Lack of Tlr 3 does not affect overall development of DRG neurons and spinal cord circuits. We asked whether itch deficiency is a result of abnormal development of the DRGs and spinal cords in $\mathrm{Trr}^{-/-}$mice. First, we analyzed the size-distribution profile of DRG neurons using the pan-neuronal marker SCG-10 and found no evidence of cell loss or size changes (Supplemental Figure 4A). Second, $\mathrm{Tlr}^{-/ /}$mice exhibited normal expression patterns of the neurochemical markers TRPV1, CGRP, P2X3, and NF200 in DRGs (Supplemental Figure 4B). Third, the expression of the neuronal markers NeuN and PKC $\gamma$ and the innervations of the primary afferents (CGRP/IB4) in 
A Single-cell RT-PCR in small-sized DRG neurons

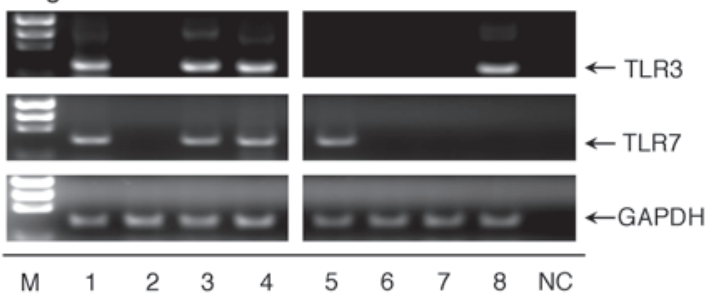

C
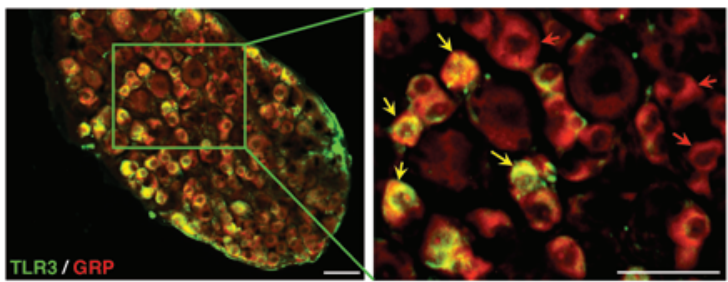

E
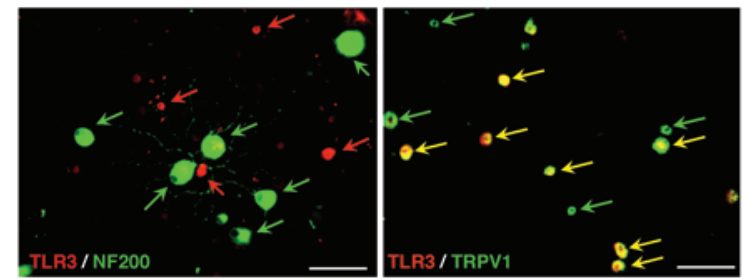

\section{B}
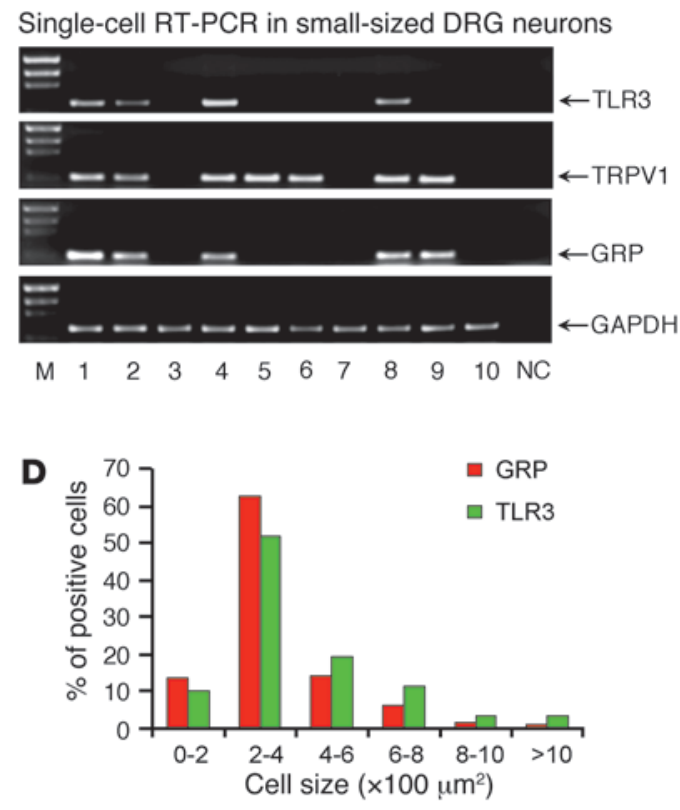

$\mathbf{F}$

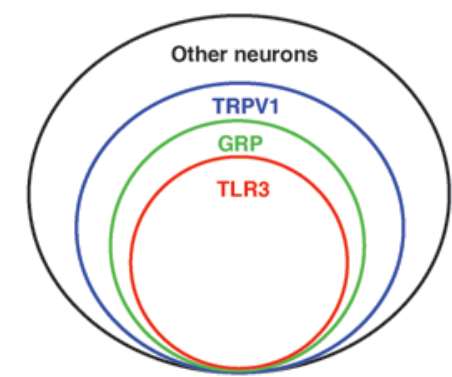

\section{Figure 4}

Expression of TLR3 in a subset of small-sized DRG neurons. (A) Single-cell RT-PCR analysis from dissociated small-sized DRG neurons showing the distinct and overlapped distribution patterns of TLR3 and TLR7 in DRG neurons. The lanes were run on the same gel but were noncontiguous. M, marker; NC, negative control. (B) Single-cell RT-PCR analysis from dissociated small-sized DRG neurons showing colocalization of TLR3 with TPRV1 and GRP. Similar results were obtained from 3 independent experiments in 30 cells collected from different animals. (C) Double immunostaining in DRGs showing co-colocalization of TLR3 and GRP. Red and yellow arrows indicate GRP+ only and double-labeled neurons, respectively. Scale bars: $50 \mu \mathrm{m}$. (D) Cell size distribution frequency of TLR3 ${ }^{+}$and GRP+ neurons. (E) Double immunostaining in cultured DRG neurons showing co-colocalization of TLR3 with TRPV1 but not with NF200. Green arrows indicate NF200+ or TRPV1+ neurons, red arrows indicate $\mathrm{TLR}^{+}$neurons, and yellow allows indicate double-labeled neurons. Scale bars: $50 \mu \mathrm{m}$. (F) A Venn diagram showing the relationship of TLR3+, GRP+, and TRPV1+ populations in a DRG. Note that all TLR3+ cells also express GRP and TRPV1.

the spinal dorsal horn were also indistinguishable between WT and $\mathrm{Tlr}^{-/-}$mice (Supplemental Figure 4C). Fourth, we examined nerve innervations in the hairy and glabrous skin of $\mathrm{Tlr}^{-/-}$mice. Staining with PGP9.5, a pan-neuronal marker, in both hairy and paw glabrous skin revealed comparable nerve innervations in WT and $\mathrm{Tlr}^{-/-}$mice (Supplemental Figure 5A). The skin innervations of the large A-fiber axons $\left(\mathrm{NF}_{200^{+}}\right)$and small peptidergic axons $\left(\mathrm{CGRP}^{+}\right)$ were also normal in $\mathrm{Tlr}^{-/-}$mice (Supplemental Figure 5, B and C). Finally, real-time RT-PCR analysis showed the normal expression of the other TLR family members (TLR2, TLR4, and TLR7) in DRGs of $\mathrm{Tlr}^{-/-}$mice (data not shown). Thus, it is unlikely that the itch deficiency that we observed in the $T l 3^{-/-}$mice resulted from the developmental defects in DRGs and spinal cords.

Algesic or pruritic agents induce normal responses in DRG neuronal somata of $\mathrm{Tlr}^{-/-}$mice. We used $\mathrm{Ca}^{2+}$ imaging to investigate the responsiveness of DRG neuronal cell bodies to algesic and pru- ritic agents, including capsaicin, histamine, and CQ. In WT mice, capsaicin, histamine, and CQ induced intracellular $\mathrm{Ca}^{2+}$ increases in $39 \%, 10 \%$, and $5 \%$ of neurons, respectively, in agreement with a previous observation (19). Of note, these $\mathrm{Ca}^{2+}$ responses were the same as those in $\mathrm{Tlr}^{-/-}$mice (Supplemental Figure 6). Consistently, the amplitude of capsaicin-induced currents was comparable between WT and Tlr3 ${ }^{-1-}$ mice (Figure 5A). Thus, TLR3 deficiency does not alter responsiveness of DRG neurons to the frequently used algesic or pruritic agents in mice.

TLR3 is critically involved in spinal cord excitatory synaptic transmission. As DRG neurons project centrally to the spinal cord, we asked whether TLR3 can be transported to the spinal central terminals. Double staining demonstrated that TLR3 was expressed in both the TRPV1containing dorsal root axons and SP-containing primary afferent terminals in the dorsal horn (Supplemental Figure 7, A and B). Next, we performed patch-clamp recordings in lamina II neurons of spinal 
A

WT

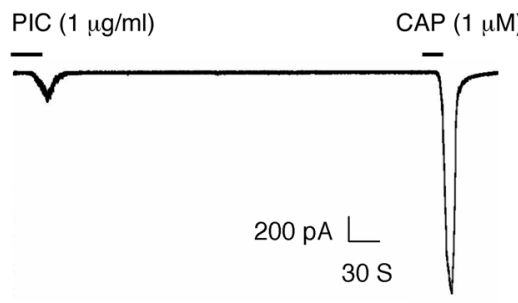

C

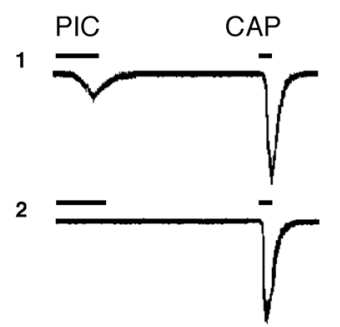

3
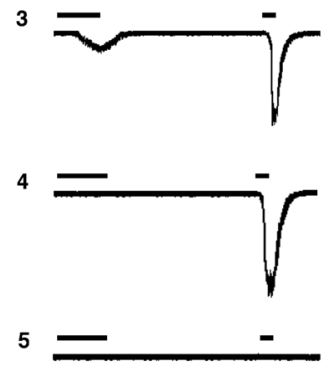

7
TIr3

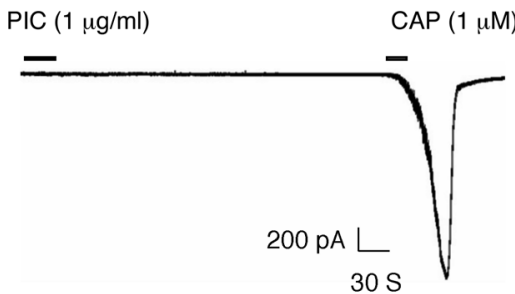

B

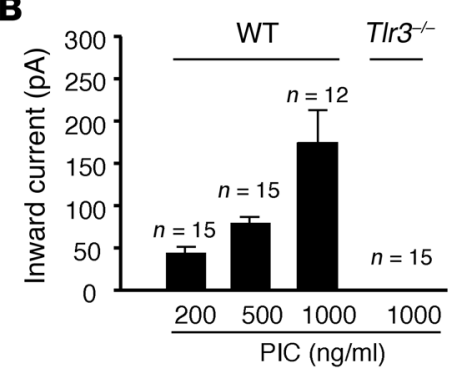

D
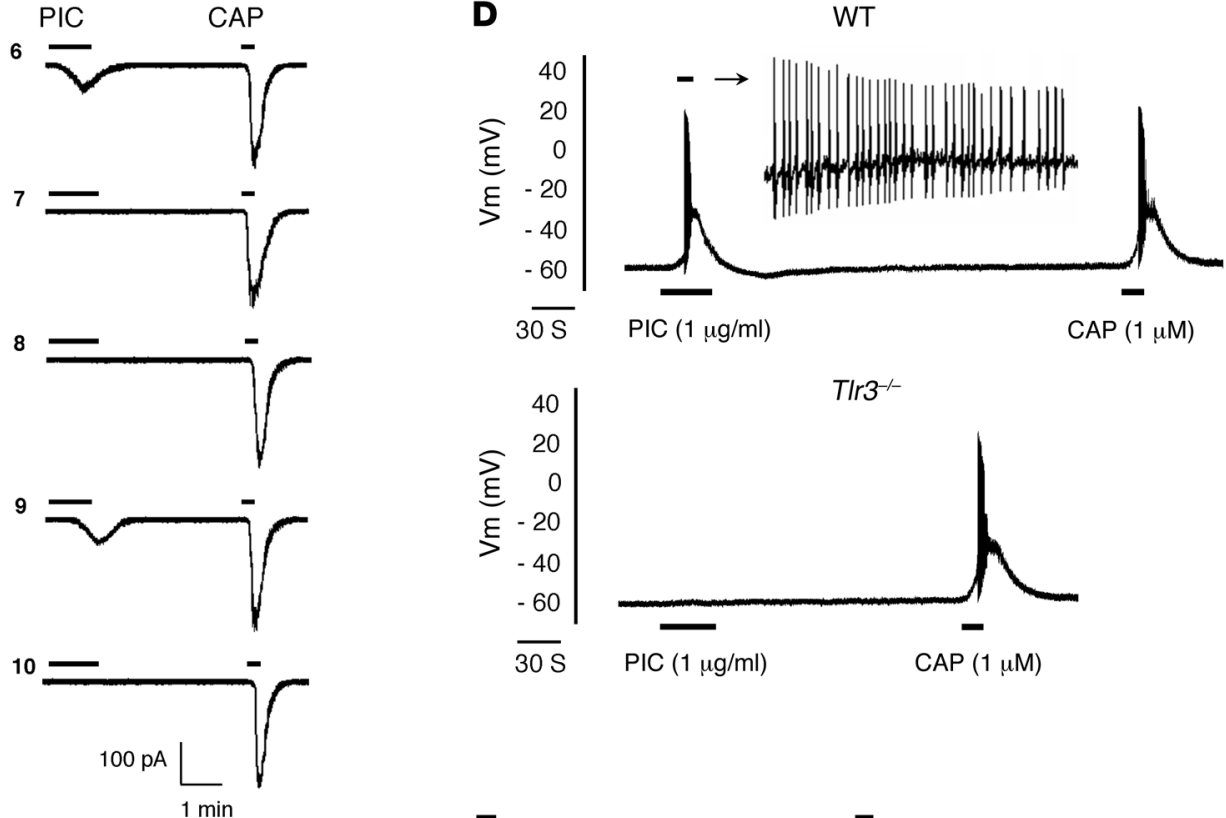

$\begin{array}{llllllllllll}1 & 2 & 3 & 4 & 5 & 6 & 7 & 8 & 9 & 10 & \mathrm{NC}\end{array}$
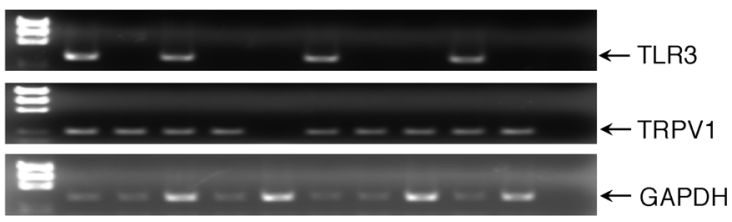

E
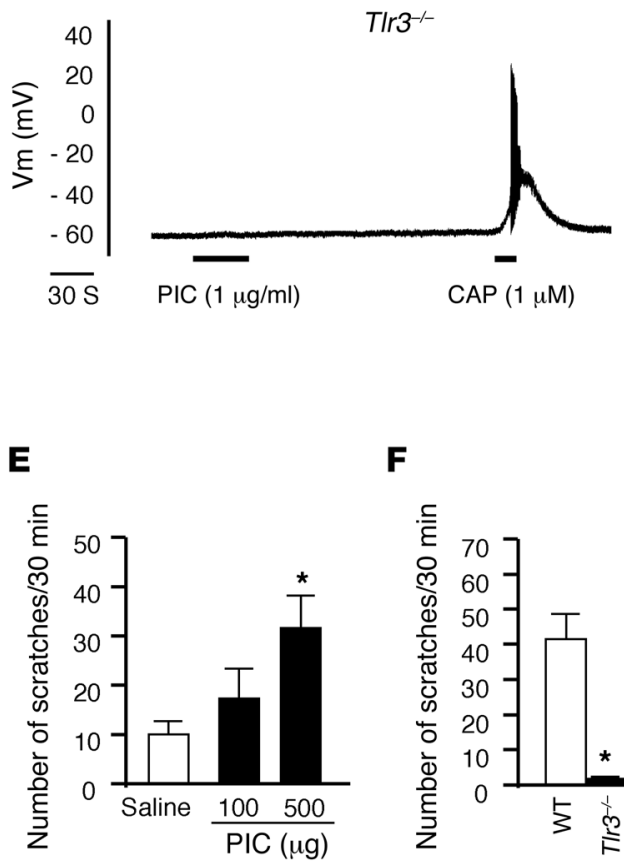

F
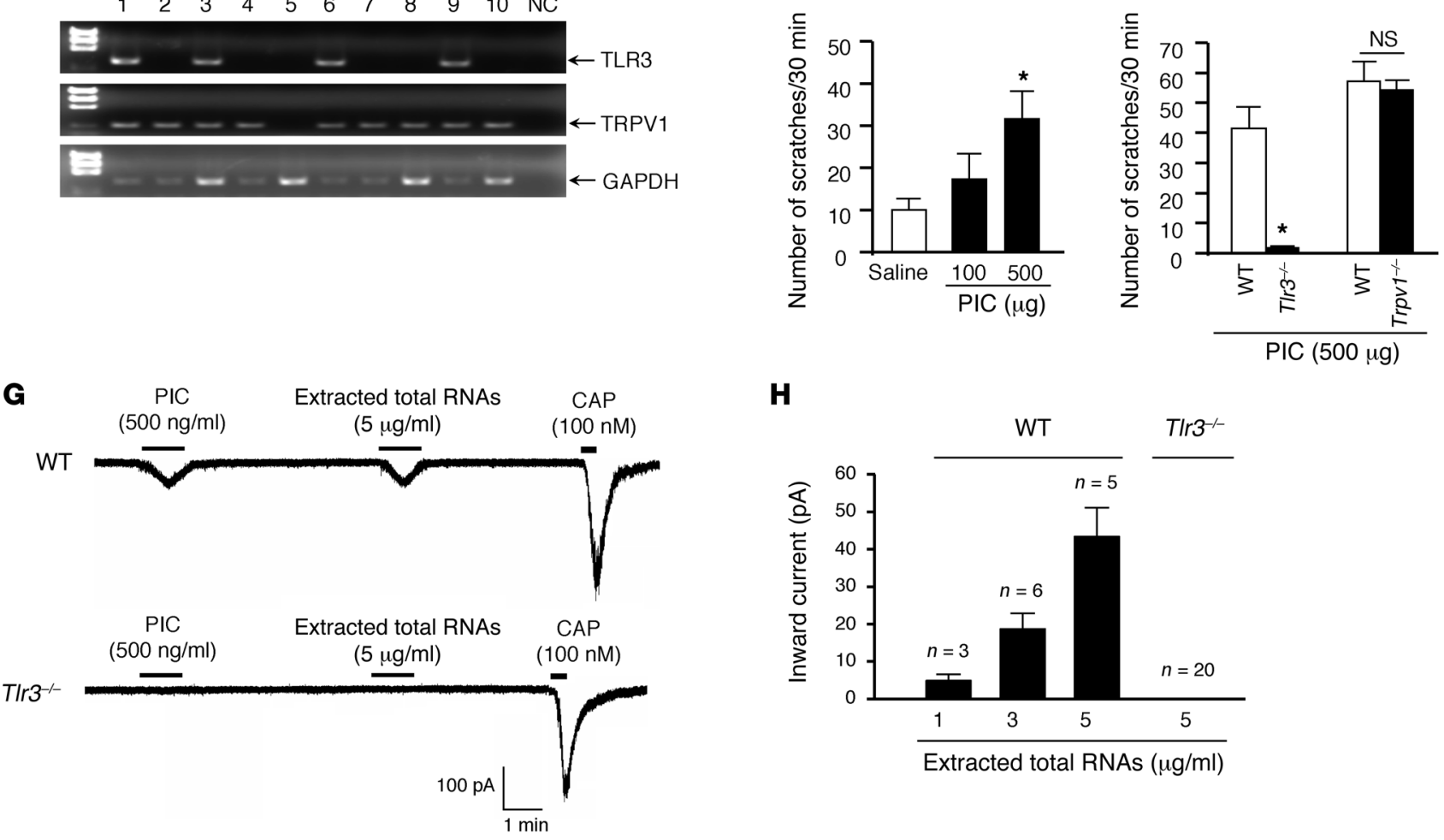

H

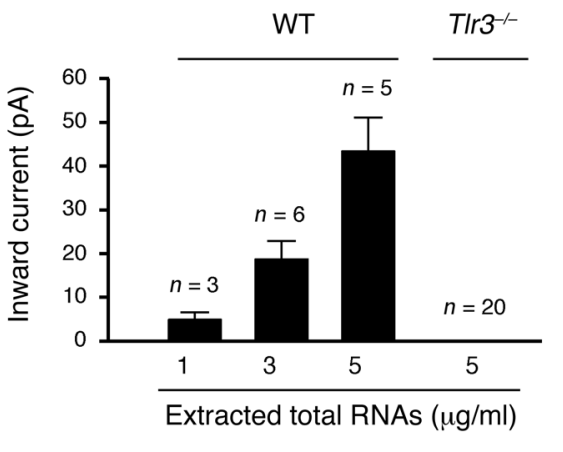




\section{Figure 5}

PIC induces inward current and action potentials in dissociated DRG neurons and elicits scratching in WT mice via TLR3 activation. (A) Inward currents evoked by PIC and capsaicin (CAP) in dissociated small-sized DRG neurons from WT and TIr $3^{-/-}$mice. Note that PIC fails to induce inward currents in T/r3 $3^{-/-}$mice. (B) Dose-dependent inward currents induced by PIC. The number of responsive neurons is indicated on the top of each bar. (C) A combination of patch-clamp recording and single-cell RT-PCR in small-sized DRG neurons shows that all 4 neurons (out of 10$)$ that respond to PIC $(200 \mathrm{ng} / \mathrm{ml})$ also express TIr3 mRNA. (D) Action potentials evoked by PIC and capsaicin in DRG neurons from WT and TIr3 ${ }^{-1-}$ mice. Note that PIC does not induce action potentials in TIr $3^{-/}$mice ( $n=18$ neurons). (E) Intradermal PIC induces dose-dependent scratching in WT mice. ${ }^{*} P<0.05$, compared with vehicle; $n=6$ mice. $(\mathbf{F})$ Intradermal PIC induces scratching in WT mice but not $T / r 3^{-/-}$mice. ${ }^{*} P<0.05$, compared with WT, Student's $t$ test; $n=6$ mice. (G) Inward currents evoked by the extracted total RNAs, PIC, and capsaicin in small-sized DRG neurons from WT and TIr $3^{-l-}$ mice. (H) Dose-dependent inward currents induced by the total RNAs. The number of responsive neurons is indicated on the top of each bar. All the data are mean \pm SEM.

cord slices to investigate whether TLR3 would modulate glutamatergic neurotransmission. Notably, PIC increased the frequency but not the amplitude of spontaneous excitatory postsynaptic currents (sEP$\mathrm{SCs}$ ) in the lamina II neurons of WT mice but not $\mathrm{Tlr}^{-/-}$mice (Figure 6 , A and B; $P<0.05$, Student's $t$ test), as a result of TLR3-mediated glutamate release from the presynaptic terminals (30).

Since the central terminals of TRPV1-expressing nociceptors are indispensable for itch (29), we also examined whether TLR3 deficiency could cause functional deficits in the spinal central terminals. Application of capsaicin to spinal cord slices induced a dramatic increase of sEPSC frequency in lamina II neurons of WT mice, but this increase was abrogated in $T l r 3^{-/}$mice (Figure 6, C and D). In parallel, the intensity of TRPV1-expressing axons/terminals in the superficial dorsal horn was significantly reduced in $\mathrm{Tlr}^{3^{-/}}$mice (Supplemental Figure 8, A and B; $P<0.05$, Student's t test). Collectively, these data point to a specific deficit of TRPV1 signaling in the spinal central terminals but not in the cell bodies and peripheral terminals of DRG neurons in $\mathrm{Tlr}^{-{ }^{--}}$mice. In sharp contrast, basal synaptic transmission (sEPSC) and capsaicin-evoked enhancement of sEPSC were normal in $\mathrm{Tlr}^{-/-}$mice (Figure 6, E and F).

We also assessed whether spinal injection of PIC or capsaicin would elicit TLR3-dependent behaviors. Intrathecal PIC evoked a dose-dependent licking behavior (Figure 6G; $P<0.05$, 1-way ANOVA), which was abolished in $T l r 3^{-/-}$mice (Figure $6 \mathrm{H} ; P<0.05$, Student's $t$ test). Intrathecal capsaicin also induced biting, licking, and scratching behaviors in WT mice, which were substantially reduced in $T l r 3^{-/-}$mice (Figure 6I; $P<0.05$, Student's $t$ test). Despite a mild decrease in baseline sEPSC in Trpv1 $1^{-/}$mice, PIC was still able to increase spinal sEPSC in these mice (Supplemental Figure 9, A and B). Thus, TLR3 signaling is largely TRPV1 independent, although TRPV1 signaling requires TLR3 in the spinal cord.

Spinal LTP is a unique form of synaptic plasticity (16) and central sensitization (14). Tetanic stimulation of the C-fibers of the sciatic nerve elicited LTP of the field potential in WT mice (Figure 6J). Strikingly, spinal LTP could not be induced in $\mathrm{Tlr}^{-/-}$mice (Figure 6J; $P<0.05$, 2-way repeated-measures ANOVA). By contrast, spinal LTP was fully induced and maintained in $T l r 7^{-1-}$ mice (Figure 6K). These results further support a distinct role of TLR3 and TLR7 in modulating central sensitization.
Given a critical role of spinal GRP/GRP receptor signaling in pruritus $(28,31)$, we compared the spinal GRP expression in WT and Tlr $3^{-/-}$mice. As previously reported (28), GRP is expressed in primary afferent axons and terminals in the superficial dorsal horn of WT mice, but this expression was reduced in $T l r 3^{-/-}$mice (Supplemental Figure 8, A and B; $P<0.05$, Student's $t$ test). Of interest, spinal administration of $\mathrm{GRP}_{18-27}$ rescued the itch deficits in $\mathrm{Tlr} 3^{-/-}$mice and elicited comparable scratching in $\mathrm{Tlr}^{-/-}$and WT mice (Supplemental Figure 8C), indicating that the ascending itch pathway from the secondary order spinal neurons to the brain is still intact in $\mathrm{Tlr}^{-/-}$mice. Therefore, itch deficiency in $T / r 3^{-/-}$mice may be attributed to impairment in the TRPV1- and GRP-mediated neurotransmission in the spinal cord.

TLR3 in DRGs is required for the full expression of itch in adult mice. To validate our findings in the transgenic mice, we also used antisense oligodeoxynucleotides (AS-ODNs) to knock down the TLR3 expression in DRGs of adult WT mice. Intrathecal injections of the TLR3 AS-ODNs led to a partial knockdown of TLR3 protein and mRNA levels in DRGs $(P<0.05$, Student's $t$ test), without changing the TLR4 expression (Figure 7, A and B). Notably, TLR3 AS-ODNs significantly inhibited the compound 48/80- and CQ-induced scratching (Figure 7B; $P<0.05$, Student's $t$ test), without effects on thermal sensitivity (Figure $7 \mathrm{C}$ ). Similarly, intrathecal injections of the selective siRNA targeting TLR3 also decreased the expression of Tlr3, but not that of Tlr7 and $\operatorname{Tr} 9$, in DRGs and further inhibited CQ-induced scratching (Figure 7, D and E).

Since TLR3 signals exclusively through the adaptor protein TIR domain-containing adaptor-inducing interferon- $\beta$ (TRIF) (4), we also tested the effects of a membrane-permeable peptide inhibitor of TRIF (32). Intrathecal TRIF inhibitor suppressed the scratching induced by both compound $48 / 80$ and CQ (Figure 7F; $P<0.05$, Student's $t$ test). These results suggest that TLR3 signaling in DRGs is also important for the full expression of histaminedependent and -independent itch in adult WT mice that do not have the genetic complications of the $T l r 3^{-/-}$mice.

TLR3 plays an essential role in dry skin-induced chronic pruritus. Chronic itch is often associated with skin diseases such as atopic dermatitis, contact dermatitis, and xerosis (dry skin) in humans (33). We further investigated the involvement of TLR3 in a dry skin-induced chronic itch condition.

As the first step, we examined whether the skin of $\mathrm{Tlr}^{-/-}$mice exhibit morphological and biochemical changes. Histological evaluation did not reveal obvious changes in the thickness of epidermis and dermis (Supplemental Figure 10, A-D). Also, the number of hair follicles and the percentile of hair follicles at different growth stages (anagen/catagen/telogen) remained unchanged (Supplemental Figure 10, B and C).

Given a crucial role of skin mast cells in pruritus, we also checked the number and functions of mast cells in $\mathrm{Tlr} 3^{-/-}$mice. We found that the number of mast cells in hairy skin was unchanged in Tlr $3^{-/-}$mice (Supplemental Figure 10, D and E). ELISA analysis revealed that the expression and release of histamine in response to compound 48/80 was similar in the skin of WT and $T l r 3^{-/-}$mice (Supplemental Figure 10, F and G). The expression of other mast cell markers, such as mouse tryptase $\alpha / \beta 1$ (TPSAB1), chymases (CMA), c-Kit (CD117), and nerve growth factor (NGF), was also unaltered in skin of $\mathrm{Tlr}^{-/-}$mice compared with that in WT mice (Supplemental Figure 10H). 
A WT

Basal

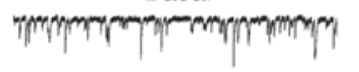

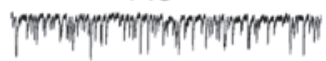

PIC

Basal TIr3

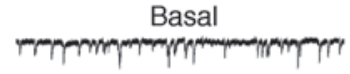

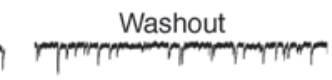

C WT

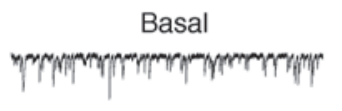

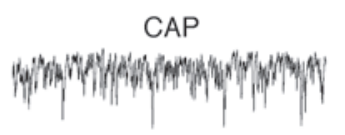

Basal

Tlr3

CAP

Washout

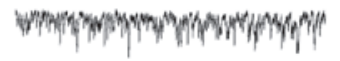

CAP WT Basal

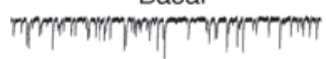

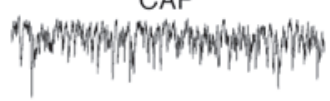

CAP

TIr ${ }^{\prime \prime}$

Basal

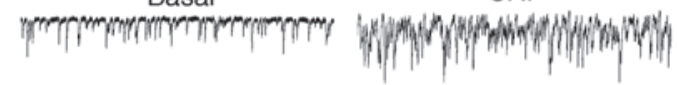

G

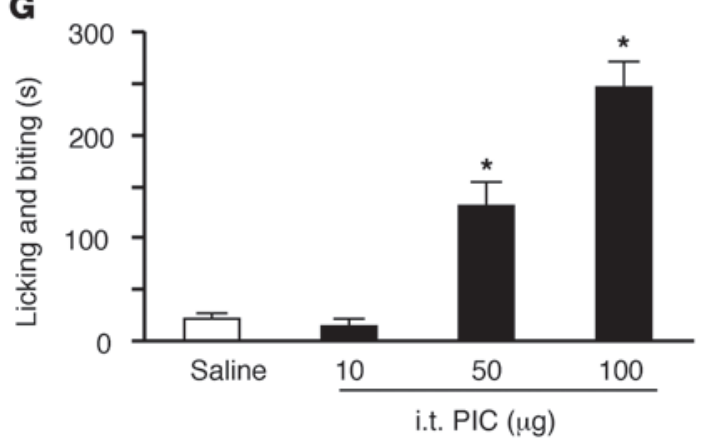

J

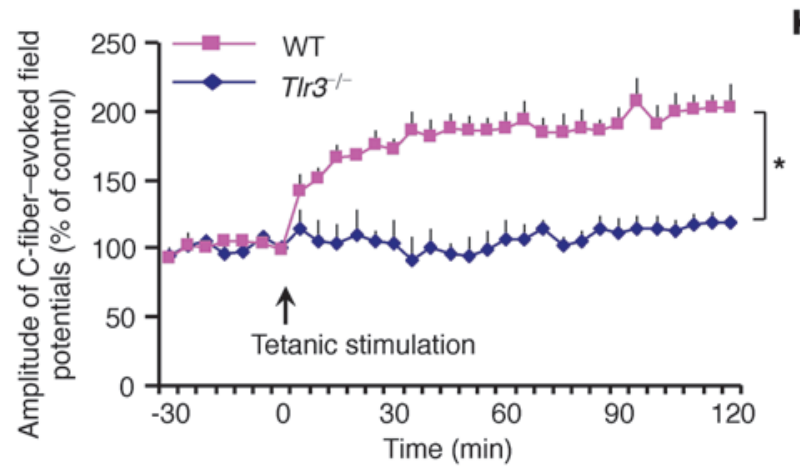

Washout

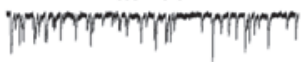
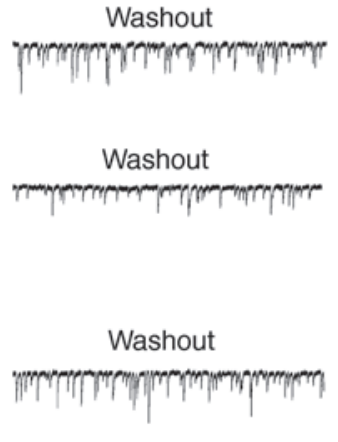

Washout

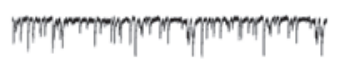
$10 \mathrm{pA} \mathrm{L} \mathrm{L}$
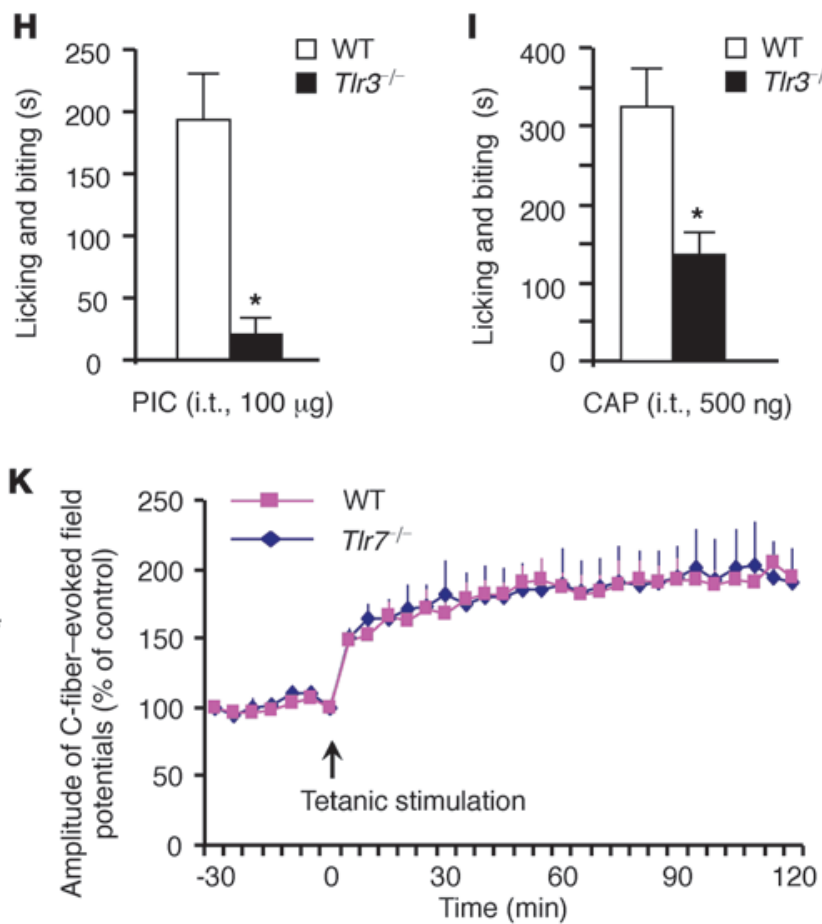

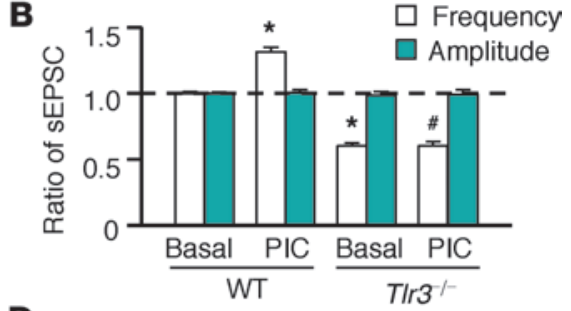

D

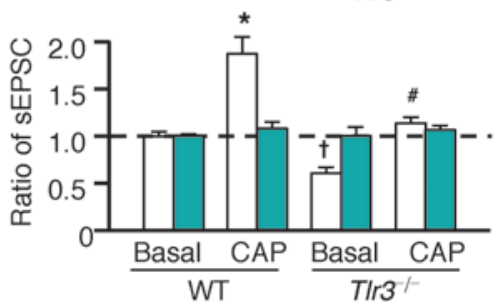

$\mathbf{F}$

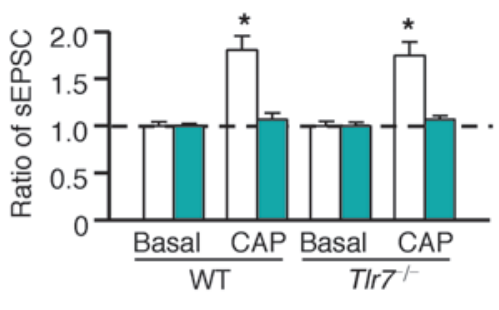

I

Figure 6

Impaired synaptic transmission and LTP induction in the spinal cord dorsal horn of TIr3 ${ }^{-1-}$ mice but not TIr7 $7^{-/-}$mice. (A-F) Patch-clamp recording of sEPSCs in lamina II neurons in spinal cord slices. (A, C, and E) Traces of sEPSCs in spinal cord slices of WT and TIr3-/- mice after (A) PIC $(100 \mathrm{ng} / \mathrm{ml})$ and $(\mathbf{C})$ capsaicin $(1 \mu \mathrm{M})$ treatment and $(\mathbf{E})$ of WT and $T / r 7^{-/-}$mice after capsaicin treatment $(1 \mu \mathrm{M})$. (B, D, and F) sEPSC frequency and amplitude after the same treatments in A, C, and E. PIC and capsaicin only increase the frequency but not amplitude of sEPSC frequency in WT mice but not in TIr $3^{-1-}$ mice. ${ }^{*} P<0.05,{ }^{\dagger} P<0.05$, compared with WT pretreatment baseline; ${ }^{*} P<0.05$, compared with WT-PIC or WT-capsaicin group, Student's $t$ test; $n=5-6$ neurons. (G) Intrathecal (i.t.) PIC induces dose-dependent licking and biting in WT mice. ${ }^{*} P<0.05$, Student's $t$ test, compared with vehicle; $n=6$ mice. (H) Intrathecal PIC or (I) capsaicin induces licking and biting in WT mice but not $T / r 3^{-1-}$ mice. ${ }^{\star} P<0.05$, Student's $t$ test, compared with WT, $n=6$ mice. ( $\mathbf{J}$ and $\mathbf{K})$ In vivo recordings of LTP of C-fiber-evoked filed potentials in the spinal cords of ( $\mathbf{J})$ TIr $3^{-/-}$and (K) TIr $7^{-/-}$mice and their corresponding WT controls. Note that spinal LTP is induced in TIr $7^{-/-}$mice but not in TIr3 ${ }^{-/-}$mice. ${ }^{*} P<0.05$, compared with WT mice, 2-way repeated-measures ANOVA; $n=5$ mice. All the data are mean \pm SEM. 
A Western blotting

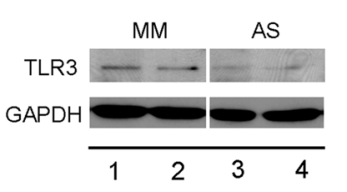

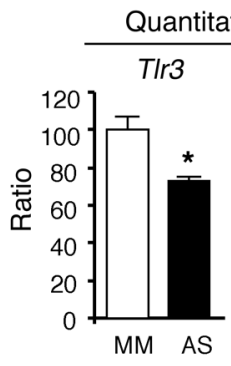

uantitative RT-PCR

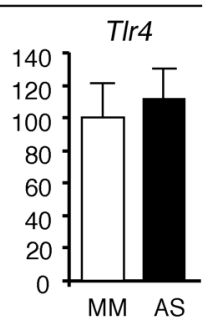

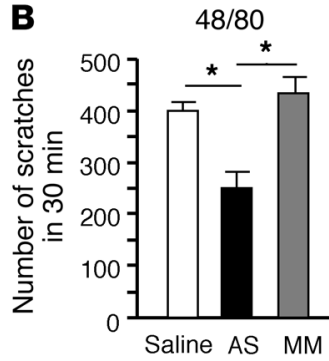

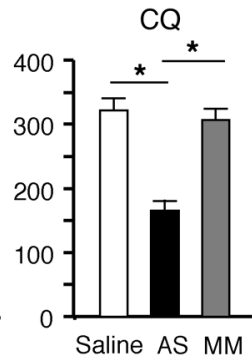

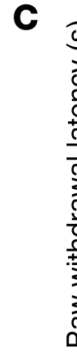

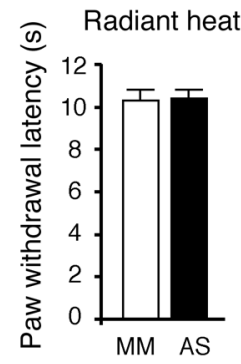

D

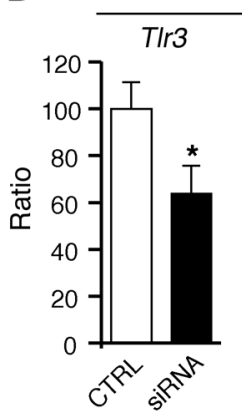

Quantitative RT-PCR

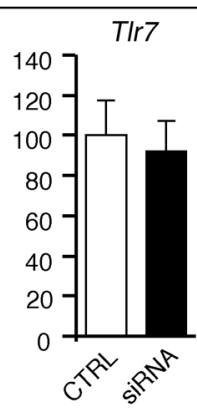

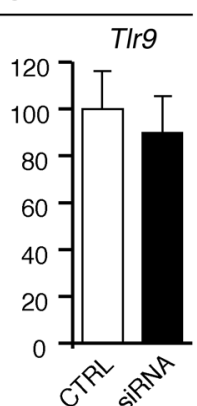

E

$C Q$

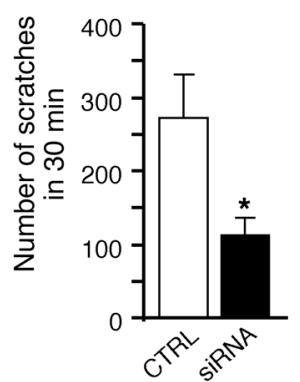

$\mathbf{F}$
$48 / 80$

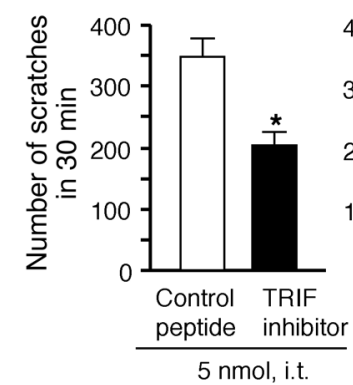

$\mathrm{CQ}$

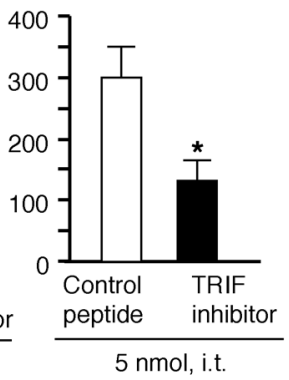

Figure 7

TLR3 signaling in DRGs of WT adult mice is required for pruritus. (A) Knockdown of TLR3 expression in DRGs after intrathecal injections of TIr3 AS-ODNs ( $10 \mu \mathrm{g}$ daily for 5 days). The lanes ran on the same gel but were noncontiguous. Note that both TLR3 protein and mRNA levels but not TIr4 mRNA levels in DRGs are decreased after AS-ODN treatment, revealed by Western blotting or quantitative PCR. * $P<0.05$, Student's $t$ test; $n=4$ mice. (B) Inhibition of compound 48/80- and CQ-induced scratching after treatment with TLR3 AS-ODNs. MM, mismatch oligodeoxynucleotides. ${ }^{\star} P<0.05$, Student $t$ test; $n=5$ mice. (C) No effects of TLR3 AS-ODNs on basal heat sensitivity ( $n=5$ mice). (D) Quantitative PCR reveals knockdown of TIr3 but not TIr7 and TIr9 mRNA expression in DRGs after intrathecal injections of TIr3-targeting siRNA (3 $\mu$ g daily for 3 days). ${ }^{*} P<0.05$, Student's $t$ test; $n=5$ mice. (E) Inhibition of $C Q$-induced scratching after siRNA treatment. ${ }^{*} P<0.05$, Student $t$ test; $n=5$ mice. (F) Intrathecal inhibition of TLR3 signaling with a peptide inhibitor of TRIF reduces scratching in mice. ${ }^{*} P<0.05$, Student's $t$ test; $n=5$ mice. All the data are mean \pm SEM.

To determine the role of TLR3 in chronic itch, we painted back skin with acetone and diethyether followed by water (AEW) for 7 days to induce dry skin lesions in mice $(34,35)$. Seven days after AEW treatment, WT mice showed robust spontaneous scratching on day 8 and 9, but this spontaneous itch was eliminated in $T l r 3^{-/-}$ mice (Figure 8A; $P<0.05$, Student's $t$ test). Notably, AEW treatment also elicited a dramatic (25-fold) increase of TLR3 expression in the dry skin $(P<0.05$, Student's $t$ test) but not in the DRGs (Figure $8 \mathrm{~B})$. Further, AEW treatment induced marked NGF upregulation, which was TLR3 dependent (Figure 8C; $P<0.05$, Student's $t$ test). However, AEW-induced upregulation of TNF- $\alpha$ and histamine did not require TLR3 (Figure 8, D and E).

Finally, we tested whether TLR3-mediated itch could be potentiated in the dry skin condition. After AEW treatment, a low dose of PIC $(100 \mu \mathrm{g})$ markedly potentiated dry skin-induced spontaneous scratching, which was abolished in $\mathrm{Tlr}^{-/-}$mice (Figure 8F; $P<0.05$, Student's $t$ test). Thus, TLR3 and its upregulation in the dry skin contribute importantly to the induction and sensitization of chronic itch.

\section{Discussion}

As key pathogen-responsive receptors, TLRs recognize pathogenassociated molecular patterns from foreign pathogens to mediate innate immunity. TLRs are typically expressed by immune cells, and activation of TLRs results in upregulation of proinflammatory cytokines via activation of the transcription factors NF- $\kappa B$ and interferon regulatory factor-3 (4). TLR2-TLR4 are also expressed by glial cells in the CNS and contribute to neuropathic pain development through glial activation and induction of proinflammatory cytokines/chemokines in the spinal cord (7-9). In this study, we demonstrated that TLR3 is also expressed by a subset of primary sensory neurons, namely TRPV1-expressing nociceptors. Importantly, the TLR3 agonist PIC is sufficient to induce inward currents and action potentials in DRG neurons and further elicit scratching in WT mice but not $\mathrm{Tlr}^{-/-}$mice. Further, the extracted total RNAs induced inward currents in DRG neurons via TLR3. At the spinal cord level, PIC also modulated the excitatory synaptic transmission by increasing sEPSC frequency. It appears that TLR3 synthesized in DRG cell bodies could be transported to the spinal central terminals to modulate synaptic transmission (30). Thus, primary sensory neurons express TLR3 to detect foreign pathogens and endogenous ligands (e.g., dsRNAs), leading to rapid protective behaviors such as scratching.

Although itch and pain share many similarities $(1,3,36)$, increasing evidence points to distinct molecular mechanisms of itch (19, 31). Primary sensory neurons located in the trigeminal ganglion and DRG transduce itch stimuli to the CNS $(1,3)$. As the bestcharacterized itch mediator, histamine is released from mast cells and binds $\mathrm{H} 1 / \mathrm{H} 4$ receptors on skin nerve terminals to elicit itch (37) via activation of PLC $\beta 3$ and TRPV1 $(29,38)$. CQ (an agonist of sensory neuron-specific GPCR MrgprA3) and BAM8-22 (an endogenous agonist of MrgprC11) produce histamine-independent itch 
A

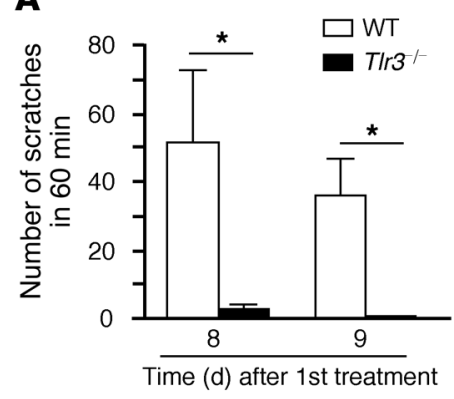

D

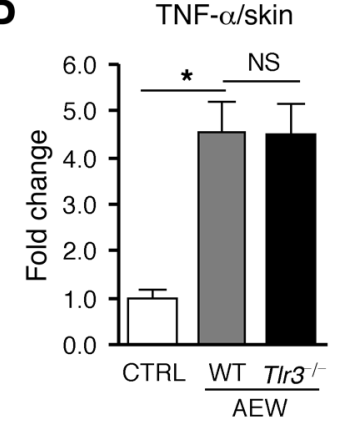

B
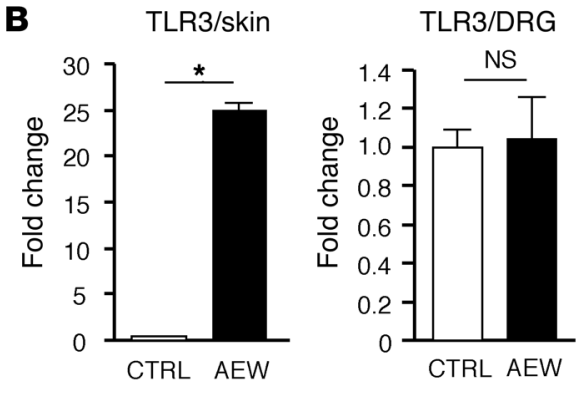

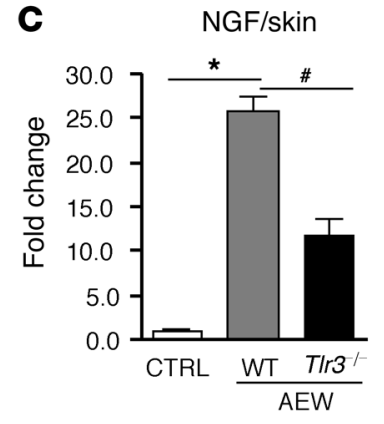

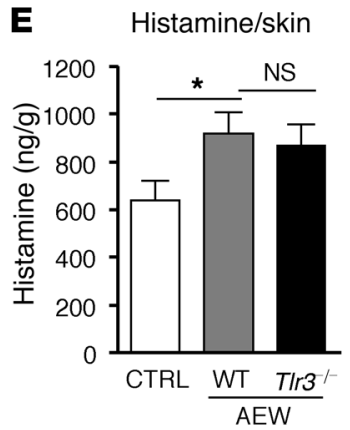

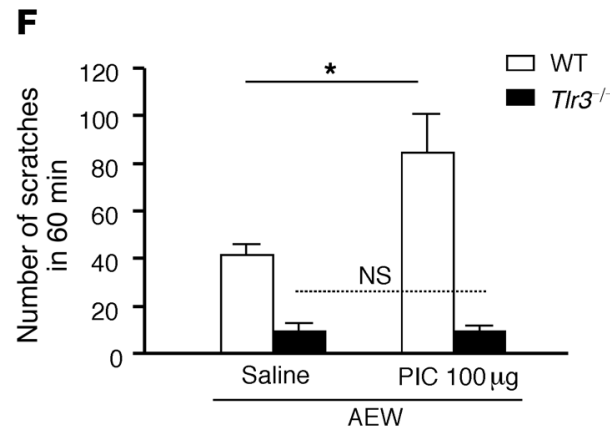

\section{Figure 8}

TLR3 is essential for dry skin-induced chronic itch. (A) Spontaneous scratching induced by acetone and diethyether (1:1) following by water (AEW, twice a day for 7 days) on day 8 and 9 in WT and TIr3-l- mice. ${ }^{*} P<0.05$. (B) Real-time quantitative RT-PCR analysis showing TLR3 upregulation in skin but not DRGs of WT mice after AEW treatment. ${ }^{*} P<0.05$. (C and D) Real-time quantitative RT-PCR analysis showing that AEW-induced upregulation of (C) NGF but not (D) TNF- $\alpha$ in the dry skin is abrogated in TIr $3^{-/-}$mice. ${ }^{*} P<0.05$, ${ }^{\#} P<0.05$. (E) ELISA analysis showing that $\mathrm{AEW}$-induced upregulation of histamine in skin is not altered in TIr3-l- mice. ${ }^{*} P<0.05$, compared with vehicle control $(\mathrm{CTRL})$. (F) Intradermal injection of PIC enhances AEW-induced spontaneous scratching, which is abrogated in TIr $3^{-/-}$mice. ${ }^{*} P<0.05$, Student's $t$ test; $n=5$ mice in all cases. All the data are mean \pm SEM.

via TRPA1 activation $(19,39)$. Both histamine-dependent itch and histamine-independent itch (e.g., itch induced by proteases, serotonin, and endothelin) require the TRPV1-expressing nociceptors $(29,40)$. Loss of vesicular glutamate transporter-2 in nociceptors results in reduced pain but enhanced itch, indicating that glutamate release from these nociceptors is dispensable for itch in the absence of pain (41). At the spinal cord level, the peptides GRP and SP, released from TRPV1-expressing nociceptors, activate GRP receptor and NK1 to elicit pruritus $(28,31,42)$. In this study, we further revealed TLR3 as a new player in itch control, to our knowledge, via its peripheral and central actions.

Of the most striking findings of this study are the marked deficits in histamine-dependent and -independent pruritus in $\mathrm{Tlr}_{\mathrm{r}} \mathrm{H}^{-1}$ mice: there was a profound reduction of scratching behaviors elicited by all the 9 pruritogens that we tested (Supplemental Figure $1 \mathrm{~K})$. Of note $\mathrm{Tlr}^{-/-}$mice showed (a) no gross anatomical defects, (b) no neuronal loss in DRGs and spinal cords, (c) no deficits in the skin and spinal cord nerve innervations, (d) no impairment in the ascending itch pathway, and (e) no changes in skin morphology. Hence, it is unlikely that the itch phenotypes that we observed are results of developmental defects in $\mathrm{Tlr}^{-/-}$mice. Consistently, knockdown of TLR3 in DRGs using AS-ODNs or siRNA and spinal inhibition of TLR3/TRIF signaling also reduced the compound 48/80- and CQ-induced itch in adult WT mice. Furthermore, direct activation of TLR 3 by intradermal PIC was sufficient to elicit TLR3-dependent scratching.
Although TLR7 was implicated in pruritus in our previous study (13), the present study has clearly demonstrated distinct roles of different TLRs in itch. Compared with a dramatic reduction in both histamine-dependent and -independent itch in $\mathrm{Tlr}^{-/-}$mice, $T l r 7^{-/-}$mice only showed a partial reduction in histamine-independent itch. Our findings also showed the limited role of TLRs in pain using the cheek model (25): the wiping behaviors induced by compound $48 / 80$ and CQ were unchanged after deletion of Tlr3 and Tlr7. However, there are at least 12 members in the mouse TLR family; the involvement of other TLRs in pain and itch needs further investigation.

Although the previous reports indicated roles of TLR3 in suppressing axonal growth of DRG neurons (12) and memory retention in the hippocampus (43), our study is the first to our knowledge to demonstrate a critical role of TLR 3 in synaptic transmission. Notably, the frequency of sEPSCs in spinal lamina II neurons was reduced in $\mathrm{Tlr}^{-/-}$mice, suggesting that TLR3 is required for glutamate release from the spinal presynaptic terminals. Consistently, PIC increased sEPSC frequency in the lamina II neurons via TLR3 activation. Despite a moderate reduction in spinal cord basal synaptic transmission, basal pain perception after thermal, mechanical, and chemical stimuli was intact in $\mathrm{Tlr}^{-/-}$mice (Figure $1, B-G)$. However, the central sensitization-induced pain hypersensitivity, i.e., the formalin-induced second-phase pain and the capsaicin-induced secondary mechanical hyperalgesia, was significantly impaired in $\mathrm{Tlr}^{-/-}$mice (Figure 1, H and I). In parallel, 
spinal cord synaptic plasticity underlying pain or itch hypersensitivity was abrogated in $\mathrm{Tlr}^{-/-}$mice. Importantly, TLR3 deficiency resulted in a failure in the induction of spinal LTP. Accordingly, the capsaicin-induced sEPSC frequency increase in dorsal horn neurons was substantially reduced in $\operatorname{Tl} 3^{-{ }^{-}}$mice (Figure 6). In sharp contrast, $\mathrm{Tl} r 7^{-/-}$mice displayed normal spinal cord synaptic transmission and LTP induction (Figure 6) and unaltered secondphase pain in the formalin test (13), despite a reduction in nonhistaminergic pruritus (13). Thus, impairment in spinal synaptic plasticity and central sensitization should contribute additionally to profound itch deficits in $\mathrm{Tlr} 3^{-/-}$mice.

We postulate that TLR3 expression on the DRG neuronal surface could be coupled to ion channels to induce inward currents and actions potentials, whereas TLR3 localization in intracellular compartments (endosomes) of DRG neurons (data not shown) could be involved in the trafficking and axonal transport of signaling molecules. A reduction in the axonal transport of TRPV1 and GRP, as observed in $\mathrm{Tlr}^{-/-}$mice (Supplemental Figure 8), should also contribute to deficits in central sensitization and itch. Of interest, a genome-scale functional screening reveals that the microtubule regulator stathmin is a potential ligand of TLR3 (44). Whether stathmin plays a role in itch by interacting with TLR3 is of great interest.

Central sensitization has been implicated in itch hypersensitivity. For example, scratching is greatly potentiated after loss of Bhlhb5-expressing inhibitory interneurons in the dorsal horn (23). Central sensitization appears to underlie the increasingly appreciated "population coding" hypothesis of itch (20, 45). According to this hypothesis, itch-responsive neurons are localized to a small population of C-fiber nociceptors expressing MrgprA3/ MrgprC11/GRP/SP/TRPV1. While activation of this small population of neurons with pruritogens elicits itch, activation of the larger population of nociceptors induces pain to suppress itch. Of interest, TLR3 is expressed in a subset of TRPV1+ nociceptors that contain GRP (Figure 4F). This unique distribution pattern of TLR3 may explain why acute pain is largely intact, whereas itch is impaired, in Tlr $3^{-/-}$mice. A specific role of TLR3 in central sensitization is supported by (a) failure of spinal LTP induction in $\mathrm{Tlr} 3^{-/-}$ mice and (b) the observation that intrathecal PIC- and capsaicininduced licking behaviors were abolished in $\mathrm{Tlr}^{-{ }^{--}}$mice.

Although our data support a neuronal mechanism of TLR3 for itch control, we should not exclude other mechanisms that are important in disease conditions. It is known that skin cells, such as mast cells and keratinocytes, play important roles in itch sensation in skin disease conditions (3). TLR3 is expressed by mast cells and keratinocytes, and PIC activates mast cells and keratinocytes to release the proinflammatory cytokines $(46,47)$. Although we did not find obvious morphological and biochemical changes in the skin of $\mathrm{Tlr}^{-/-}$mice under the normal conditions, we observed marked TLR3 upregulation in the dry skin condition, and this upregulation may drive chronic itch via producing the proinflammatory cytokines (3). In addition, TLR3-mediated upregulation of NGF in dry skin could also regulate chronic itch, since increased epidermal NGF expression was implied in the pathogenesis of pruritic contact dermatitis and psoriasis (48).

In conclusion, we have identified TLR3 as a critical signaling molecule that is required for pruritus, regardless of histamine dependence. Functional TLR3 is expressed by primary sensory neurons that coexpress TRPV1 and GRP, and it is known that this subset of nociceptors is indispensable for itch sensation. The TLR3 agonist PIC was sufficient to induce inward currents and action potentials in
DRG neurons, increase synaptic transmission in spinal dorsal horn neurons, and elicit scratching in WT mice. In particular, TLR3 deficiency resulted in impairment in central sensitization and spinal cord synaptic plasticity underlying itch hypersensitivity. TLR3 is further upregulated in dry skin and essential for the development of chronic itch. Given (a) the prevalence of chronic itch during skin diseases (e.g., atopic and contact dermatitis) as well as kidney, liver, and metabolic diseases and (b) the ineffectiveness of current antihistamine treatments in these chronic itchy conditions $(2,3)$, targeting TLR3 may provide a novel strategy for developing anti-itch therapies.

\section{Methods}

Additional details regarding this study are provided in the Supplemental Methods.

Mice. We used adult mice (25-32 g) for behavioral and histochemical studies. $\operatorname{Tlr}^{-/-}$mice (B6;129S1-Tlr3tm1Flv/J mice) and WT control mice of the same genetic background (B6129SF1/J mice) as well as Trpv1/-- and $T l r 7^{-/-}$mice and their WT control mice (C57BL/6J mice) were purchased from The Jackson Laboratory and bred at Harvard Thorn Building Animal Facility. To confirm the results from the transgenic mice, we also purchased adult WT CD1 mice from Charles River for some behavioral and pharmacological studies.

Behavioral analysis. All of the behavioral tests were done in strict sex- and age-matched adult mice by the observers blinded to the treatment or genotype of the animals. Mice were acclimatized to the testing environment daily for at least 2 days before the analysis. Itch and pain behavioral testing in mice was performed as previously described in detail $(13,19,28)$.

Immunohistochemistry. Immunohistochemistry was performed according to standard protocols, and the following primary antibodies were used: rabbit anti-TLR3 antibody (1:1,000; Santa Cruz Biotechnology Inc.), guinea pig anti-TRPV1 antibody (1:1,000; Neuromics), rabbit anti-GRP antibody (1:1,000; Immunostar), guinea pig anti-SP antibody (1:1,000; Neuromics), rabbit anti-Fos antibody (1:500; Santa Cruz Biotechnology Inc.), mouse anti-NF200 (1:2,000; Sigma-Aldrich), mouse anti-NeuN antibody (1:2,000; Millipore), rabbit anti-CGRP antibody (12,000; Neuromics), rabbit anti-PKC $\gamma$ antibody (1:2,000; Santa Cruz Biotechnology Inc.), rabbit anti-PGP9.5 antibody (1:1,000; Biogenesis).

Drugs and administration. HTMT and 4-MeHA were obtained from Tocris. Endothelin-1 was from ALEXIS Biochemicals. SLIGRL-NH2 and GRP1827 were from Bachem. TRIF peptide inhibitor and control peptide were from Invivogen. AS-ODNs targeting TLR3 and mismatch oligodeoxynucleotides were synthesized by Invitrogen. siRNA targeting TLR3 and nontargeting control siRNA were synthesized by Dharmacon. All other chemicals were obtained from Sigma-Aldrich. We injected a pruritic agent intradermally in the nape of the neck $(50 \mu \mathrm{l})$ or cheek $(10 \mu \mathrm{l})$ to elicit itch. We injected $20 \mu \mathrm{l}$ capsaicin, mustard oil, or formalin into the plantar surface of 1 hind paw to elicit pain. We intrathecally injected $10 \mu \mathrm{l}$ TLR3 AS-ODN (10 $\mu \mathrm{g}$, daily for 5 days), TLR3 siRNA ( $3 \mu \mathrm{g}$, daily for 3 days), TRIF inhibitor (5 nmol), GRP-peptide (1 nmol), PIC (10-100 $\mu \mathrm{g})$, or capsaicin (500 ng) via a lumbar puncture, under a brief anesthesia with isoflurane.

Single-cell RT-PCR. Single-cell RT-PCR was performed as previously described (13). A single cell was aspirated into a patch pipette with a tip diameter of about $25 \mu \mathrm{m}$, gently put into a reaction tube containing reverse transcription reagents, and incubated for 1 hour at $50^{\circ} \mathrm{C}$ (SuperScript III, Invitrogen). The cDNA product was then used in separate PCR. The sequences of all the primers used for single-cell PCR are described in Supplemental Table 1. The first- and second-round PCR was preformed using "outer" primers and "inner" primers, respectively. A negative control was obtained from pipettes that did not harvest any cell contents but were submerged in the bath solution. 
Patch-clamp recordings in DRG neurons and spinal cord slices. Whole-cell patchclamp recordings were performed at room temperature to measure inward currents (voltage clamp, at $-60 \mathrm{mV}$ holding potential) and action potentials (current clamp) in small-sized DRG neurons, using Axopatch-200B amplifier (49). Whole-cell patch-clamp recordings were also conducted in lamina II neurons in spinal cord slices (30) to measure sEPSC in a voltage clamp mode $(-70 \mathrm{mV})$. We removed a portion of the lumbar spinal cord (L4-L5) from young mice (4-6 weeks old) under urethane anesthesia $(1.5-2.0 \mathrm{~g} / \mathrm{kg}$, intraperitoneally) and cut transverse spinal cord slices $(400-600 \mu \mathrm{m})$ on a vibrating microslicer.

Spinal cord LTP recordings in anesthetized mice. Mice were anesthetized with urethane $(1.5 \mathrm{~g} / \mathrm{kg}$, intraperitoneally). After electrical stimulation of the sciatic nerve, the field potentials were recorded in the ipsilateral L4-L5 spinal segments with glass microelectrodes $100-300 \mu \mathrm{m}$ from the surface of the cord. After recording stable responses following test stimuli (2 times C-fiber threshold, 0.5 -ms period, 1-minute interval, every 5 minutes) for more than 40 minutes, conditioning tetanic stimulation (5 times C-fiber threshold, $100 \mathrm{~Hz}$, 1-s period, 4 trains, 10-s interval) was delivered to the sciatic nerve for inducing LTP of C-fiber-evoked field potentials in the spinal cord, as we previously reported (49).

Real-time quantitative RT-PCR. We collected lumbar and cervical DRGs from WT and $\mathrm{Tlr}^{-/-}$mice and isolated total RNAs using the RNeasy Plus Mini Kit (Qiagen). One microgram of RNA was reverse transcribed for each sample using Omniscript reverse transcriptase (Qiagen). Sequences for all the forward and reverse primers are described in Supplemental Table 2. Triplicate qPCR analyses were performed using the Opticon Real-Time PCR Detection System (Bio-Rad).

Histamine measurement. Histamine levels from the skin of normal and AEW-treated WT and $\mathrm{Tlr}^{-/-}$mice were quantified using a histamine Enzyme Immunoassay Kit (Oxford Biomedical Research). Histamine release was also measured in skin organ cultures after treatment of compound $48 / 80$.

Western blotting. DRGs were rapidly removed and homogenized in a lysis buffer containing a cocktail of protease inhibitors and phosphatase inhibitors. The protein concentrations were determined by a BCA Protein Assay
(Pierce), and $30 \mu \mathrm{g}$ of proteins were loaded for each lane and separated on SDS-PAGE gel (4\%-15\%; Bio-Rad). After the transfer, the blots were incubated overnight at $4{ }^{\circ} \mathrm{C}$ with polyclonal antibody against TLR-3 (rabbit, 1:500; Imgenex) and GAPDH (rabbit, 1:10,000; Sigma-Aldrich).

Statistics. All the data are expressed as mean \pm SEM. Most data were analyzed with 2-tailed Student's $t$ test (such as 2 groups from WT and KO mice). One-way ANOVA followed by post-hoc Bonferroni test was used for multiple comparisons. Two-way repeated-measures ANOVA was also used to analyze the data with multiple time points (e.g., time course of itch behaviors and spinal cord LTP). The criterion for statistical significance was $P<0.05$. Supplemental Table 3 shows an example of quantification of immunohistochemistry in DRG sections.

Study approval. All animal procedures performed in this study were reviewed and approved by the Harvard Medical Area Standing Committee on Animals. The experiments were conducted in accordance with the $\mathrm{NIH}$ guidelines for care and use of animals and the recommendations of International Association for the Study of Pain.

\section{Acknowledgments}

This work was supported by US NIH grants R01-DE17794, R01NS54362, and R01-NS67686 to R.-R. Ji.

Received for publication November 4, 2011, and accepted in revised form March 21, 2012.

Address correspondence to: Ru-Rong Ji, Pain Research Division, Department of Anesthesiology, Duke University Medical Center, DUMC Box 3094, GSRB-I, Room 1027A, 595 LaSalle Street, Durham, North Carolina 27710, USA. Phone: 919.684.9387; Fax: 919.681.4776; E-mail: ru-rong.ji@duke.edu.

Tong Liu, Temugin Berta, Zhen-Zhong Xu, Chul-Kyu Park, and Ru-Rong Ji's present address is: Pain Research Division, Department of Anesthesiology, Duke University Medical Center, Durham, North Carolina, USA.
1. Ikoma A, Steinhoff M, Stander S, Yosipovitch G, Schmelz M. The neurobiology of itch. Nat Rev Newrosci. 2006;7(7):535-547.

2. Yosipovitch G, Greaves MW, Schmelz M. Itch. Lancet. 2003;361(9358):690-694.

3. Paus R, Schmelz M, Biro T, Steinhoff M. Frontiers in pruritus research: scratching the brain for more effective itch therapy. JClin Invest. 2006;116(5):1174-1186.

4. Kawai T, Akira S. The role of pattern-recognition receptors in innate immunity: update on Toll-like receptors. Nat Immunol. 2010;11(5):373-384.

5. Town T, Jeng D, Alexopoulou L, Tan J, Flavell RA. Microglia recognize double-stranded RNA via TLR3. J Immunol. 2006;176(6):3804-3812.

6. Wang T, Town T, Alexopoulou L, Anderson JF, Fikrig E, Flavell RA. Toll-like receptor 3 mediates West Nile virus entry into the brain causing lethal encephalitis. Nat Med. 2004;10(12):1366-1373.

7. Kim D, et al. A critical role of toll-like receptor 2 in nerve injury-induced spinal cord glial cell activation and pain hypersensitivity. J Biol Chem. 2007;282(20):14975-14983.

8. Obata K, et al. Toll-like receptor 3 contributes to spinal glial activation and tactile allodynia after nerve injury. J Neurochem. 2008;105(6):2249-2259.

9. Tanga FY, Nutile-McMenemy N, Deleo JA. The CNS role of Toll-like receptor 4 in innate neuroimmunity and painful neuropathy. Proc Natl Acad Sci US A. 2005;102(16):5856-5861.

10. Wadachi R, Hargreaves KM. Trigeminal nociceptors express TLR-4 and CD14: a mechanism for pain due to infection. J Dent Res. 2006;85(1):49-53. 11. Diogenes A, Ferraz CC, Akopian AN, Henry MA, Hargreaves KM. LPS sensitizes TRPV1 via activation of TLR4 in trigeminal sensory neurons. J Dent Res. 2011;90(6):759-764.

12. Cameron JS, et al. Toll-like receptor 3 is a potent negative regulator of axonal growth in mammals. J Neurosci. 2007;27(47):13033-13041.

13. Liu T, Xu ZZ, Park CK, Berta T, Ji RR. Tolllike receptor 7 mediates pruritus. Nat Neurosci. 2010;13(12):1460-1462.

14. Ji RR, Kohno T, Moore KA, Woolf CJ. Central sensitization and LTP: do pain and memory share similar mechanisms? Trends Neurosci. 2003;26(12):696-705.

15. Patel KN, Dong X. An itch to be scratched. Neuron. 2010;68(3):334-339.

16. Ruscheweyh R, Wilder-Smith O, Drdla R, Liu XG, Sandkuhler J. Long-term potentiation in spinal nociceptive pathways as a novel target for pain therapy. Mol Pain. 2011;7:20.

17. Ji RR, Kohno T, Moore KA, Woolf CJ. Central sensitization and LTP: do pain and memory share similar mechanisms? Trends Neurosci. 2003;26(12):696-705.

18. Alexopoulou L, Holt AC, Medzhitov R, Flavell RA. Recognition of double-stranded RNA and activation of NF-kappaB by Toll-like receptor 3. Nature. 2001;413(6857):732-738.

19. Liu Q, et al. Sensory neuron-specific GPCR Mrgprs are itch receptors mediating chloroquine-induced pruritus. Cell. 2009;139(7):1353-1365.

20. Akiyama T, Merrill AW, Carstens MI, Carstens E.
Activation of superficial dorsal horn neurons in the mouse by a PAR-2 agonist and 5-HT: potential role in itch. J Neurosci. 2009;29(20):6691-6699.

21. Steinhoff M, et al. Proteinase-activated receptor2 mediates itch: a novel pathway for pruritus in human skin. J Neurosci. 2003;23(15):6176-6180.

22. Liu Q, et al. The distinct roles of two GPCRs, MrgprC11 and PAR2, in itch and hyperalgesia. Sci Signal. 2011;4(181):ra45.

23. Ross SE, et al. Loss of inhibitory interneurons in the dorsal spinal cord and elevated itch in Bhlhb5 mutant mice. Neuron. 2010;65(6):886-898.

24. Kim SJ, et al. Analysis of cellular and behavioral responses to imiquimod reveals a unique itch pathway in transient receptor potential vanilloid 1 (TRPV1)-expressing neurons. Proc Natl Acad Sci U S A. 2011;108(8):3371-3376.

25. Shimada SG, LaMotte RH. Behavioral differentiation between itch and pain in mouse. Pain. 2008;139(3):681-687.

26. Akiyama T, Carstens MI, Carstens E. Facial injections of pruritogens and algogens excite partly overlapping populations of primary and secondorder trigeminal neurons in mice. J Neurophysiol. 2010;104(5):2442-2450.

27. Cameron JS, et al. Toll-like receptor 3 is a potent negative regulator of axonal growth in mammals. J Neurosci. 2007;27(47):13033-13041.

28. Sun YG, Chen ZF. A gastrin-releasing peptide receptor mediates the itch sensation in the spinal cord. Nature. 2007;448(7154):700-703. 
29. Imamachi N, et al. TRPV1-expressing primary afferents generate behavioral responses to pruritogens via multiple mechanisms. Proc Natl Acad Sci US A. 2009;106(27):11330-11335.

30. Kawasaki Y, Zhang L, Cheng JK, Ji RR. Cytokine mechanisms of central sensitization: distinct and overlapping role of interleukin-1beta, interleukin6 , and tumor necrosis factor-alpha in regulating synaptic and neuronal activity in the superficial spinal cord. J Neurosci. 2008;28(20):5189-5194.

31. Sun YG, Zhao ZQ, Meng XL, Yin J, Liu XY, Chen ZF. Cellular basis of itch sensation. Science. 2009;325(5947):1531-1534.

32. Toshchakov VU, Basu S, Fenton MJ, Vogel SN. Differential involvement of BB loops of toll-IL-1 resistance (TIR) domain-containing adapter proteins in. J Immunol. 2005;175(1):494-500.

33. Steinhoff M, Cevikbas F, Ikoma A, Berger TG. Pruritus: management algorithms and experimental therapies. Semin Cutan Med Surg. 2011;30(2):127-137.

34. Miyamoto T, Nojima H, Shinkado T, Nakahashi $\mathrm{T}$, Kuraishi Y. Itch-associated response induced by experimental dry skin in mice. Jpn J Pharmacol. 2002;88(3):285-292.

35. Akiyama T, Carstens MI, Carstens E. Enhanced scratching evoked by PAR-2 agonist and 5-HT but not histamine in a mouse model of chronic dry skin itch. Pain. 2010;151(2):378-383.

36. Yosipovitch G, Carstens E, McGlone F. Chronic itch and chronic pain: Analogous mechanisms. Pain. 2007;131(1-2):4-7.

37. Shim WS, Oh U. Histamine-induced itch and its relationship with pain. Mol Pain. 2008;4:29.

38. Han SK, Mancino V, Simon MI. Phospholipase Cbeta 3 mediates the scratching response activated by the histamine $\mathrm{H} 1$ receptor on C-fiber nociceptive neurons. Neuron. 2006;52(4):691-703.

39. Wilson SR, et al. TRPA1 is required for histamineindependent, Mas-related G protein-coupled receptor-mediated itch. Nat Neurosci. 2011;14(5):595-602.

40. Mishra SK, Tisel SM, Orestes P, Bhangoo SK, Hoon MA. TRPV1-lineage neurons are required for thermal sensation. EMBO J. 2011;30(3):582-593.

41. Liu Y, et al. VGLUT2-dependent glutamate release from nociceptors is required to sense pain and suppress itch. Neuron. 2010;68(3):543-556.

42. Carstens EE, Carstens MI, Simons CT, Jinks SL. Dorsal horn neurons expressing NK-1 receptors mediate scratching in rats. Neuroreport. 2010; 21(4):303-308.
43. Okun E, et al. Toll-like receptor 3 inhibits memory retention and constrains adult hippocampal neurogenesis. Proc Natl Acad Sci U S A. 2010;107(35):15625-15630.

44. Bsibsi M, et al. The microtubule regulator stathmin is an endogenous protein agonist for TLR3. J Immunol. 2010;184(12):6929-6937.

45. Ma Q. Labeled lines meet and talk: population coding of somatic sensations. J Clin Invest. 2010;120(11):3773-3778.

46. Kulka M, Alexopoulou L, Flavell RA, Metcalfe DD. Activation of mast cells by double-stranded RNA: evidence for activation through Toll-like receptor 3. J Allergy Clin Immunol. 2004;114(1):174-182.

47. Tohyama $\mathrm{M}$, et al. dsRNA-mediated innate immunity of epidermal keratinocytes. Biochem Biophys Res Commun. 2005;335(2):505-511.

48. Botchkarev VA, et al. Neurotrophins in skin biology and pathology. J Invest Dermatol. 2006; 126(8):1719-1727.

49. Park CK, Lu N, Xu ZZ, Liu T, Serhan CN, Ji RR. Resolving TRPV1- and TNF-alpha-mediated spinal cord synaptic plasticity and inflammatory pain with neuroprotectin D1. J Neurosci. 2011;31(42):15072-15085. 